\title{
A simple empirical inventory model for managing the processed corneal tissue equitably in hospitals with demand differentiation
}

\author{
Mahnaz Sohrabi $^{1}$ (D) Mostafa Zandieh ${ }^{2}$ (D) Behrouz Afshar-Nadjafi ${ }^{1}$ (D)
}

Received: 5 February 2021 / Revised: 4 September 2021 / Accepted: 29 September 2021 /

Published online: 21 October 2021

(c) SBMAC - Sociedade Brasileira de Matemática Aplicada e Computacional 2021

\begin{abstract}
This paper peruses one of the most challenging healthcare concerns related to human organ transplantation. In this regard, a medical system's health-economic objectives are investigated to (1) achieve a desirable level of health equity in offering healthcare services to patients who are differentiated in terms of medical conditions, and (2) minimize total costs incurred across managing the inventory. This paper presents the first-ever operational research study to manage processed corneal tissue (PCT). Besides, hybrid demand-oriented policies have been proposed to equitably issue and assign specific inventories to demands with distinct medical urgent levels (ULs). In this regard, a practical multi-objective mixed-integer linear programming (MOMILP) model is proposed for a system with multiple products that can be substituted with each other according to medical priorities. A goal programming (GP) approach is utilized to find the optimum solution. The applicability of the model is validated through a real case study. Finally, several sensitivity analyses are conducted to examine the effect of critical parameters on the solutions to gain useful managerial insights. The results show that the proposed health-economic trade-off is well performed and can efficiently handle the real case.
\end{abstract}

Keywords Eye banking - Inventory management - Healthcare supply chain · Health equity . Substances of human origin

Communicated by Anibal Tavares de Azevedo.

$\bowtie \quad$ Mostafa Zandieh

m_zandieh@sbu.ac.ir

Mahnaz Sohrabi

m.sohrabi@qiau.ac.ir

Behrouz Afshar-Nadjafi

afsharnb@alum.sharif.edu

1 Faculty of Industrial and Mechanical Engineering, Qazvin Branch, Islamic Azad University, Qazvin, Iran

2 Department of Industrial Management and Information Technology, Management and Accounting Faculty, Shahid Beheshti University, GC, Tehran, Iran 
Mathematics Subject Classification 90-10 · 90B05 · 90C11 -90C29 · 90C90

\section{Introduction}

Medical products are mostly perishable with increasing demand due to the growing population (Santhi and Karthikeyan 2016). Managing the medical products in such a way as to improve the quality of health services while minimizing their wastage and shortage is always associated with some complexities (Chaudhary et al. 2018; Sohrabi et al. 2021b). Most medical products need special devices to maintain and control the temperature. Therefore, storing more stocks increases holding costs, employing storage facilities, and probably wastage cost due to expiration (Bhattacharya and Lam 2020).

Moreover, the management of some crucial perishable products, which could rarely be found, is usually expensive. It gets complicated when they have a very limited shelf life and finite supply resources (Hosseini-Motlagh et al. 2020b). For instance, one can mention blood products such as blood platelet or other transplantable organs (e.g., eye cornea) and recombinant vaccines. The main challenges are how to face the increasing demand and supply constraints of such products, taking not accessibility to donors into account, holding enough amounts of stocks for demand satisfaction, and choosing a fair inventory assignment policy to serve different urgent levels (ULs) of patients considering health equity (Sohrabi et al. 2021a). These issues should be eliminated to provide a proper health service to patients while the least possible shortage and wastage happen (Liu et al. 2020). In this regard, inventory management systems are powerful tools for improving clinical setting quality and play a vital role in healthcare supply chains (HSCs) (Santhi and Karthikeyan 2016; Chaudhary et al. 2018).

Finding efficient ways to reduce total costs and improve the operation of the HSC are two of the critical concerns of healthcare managers (Civelek et al. 2015). It is more significant for vital medical products that cannot be produced indefinitely and are processed from human organs tissues. Accordingly, the utilization of streamlined inventory management techniques can significantly impact making the healthcare system's operations more valuable to a large extent (Santhi and Karthikeyan 2016).

Healthcare systems mainly focus on delivering the best quality of service for patients. However, the increasing population age, recent medical advances, more aggressive treatment of some diseases, and the growing need for various medicines have made it challenging to provide the highest medical services and products required by the health system (Mousazadeh et al. 2018). Limited resources of healthcare systems and some rare medical products make it essential to consider health equity in delivering services to patients with different medical priorities (Sohrabi et al. 2021a). Health equity is a critical criterion in health services. How one defines health equity can have important policy implications and affect operational consequences. It can determine by which measures healthcare services performance should be monitored and qualified and which activities will receive support from resources allocated to address health equity (Braveman 2006).

Although meeting demand is a great responsibility for healthcare systems, the limited financial resources and operational cost of handling medical inventories cannot be neglected (Civelek et al. 2015). Considering practical policies to make an efficient and effective system had become a significant concern for governments and healthcare managers due to the lively growth in healthcare expenditure globally as well as demand heightening for healthcare services (Saha and Ray 2018). Thus, the performance within healthcare supply chains must 
be improved by delivering the highest possible benefits and optimizing operational costs to make the best use of limited medical and financial resources (Sohrabi et al. 2021a).

Generally, healthcare systems have two main health-economic objectives. First, to provide demand-oriented services considering health equity and making it accessible for the patients at various medical Urgent Levels (ULs) followed by shortage reduction without any wastage or failure. Second, to minimize the system's total costs by adopting cost-efficient policies (Cheraghi and Hosseini-Motlagh 2018). In this regard, a systematic inventory management approach and timely use of medical products before their expiration date can dramatically help the healthcare system spend its financial resources more economically by removing redundant costs without weakening the offered healthcare services (Rajendran and Ravindran 2019). The current study aims to find a proper solution for healthcare managers' mentioned concerns by proposing a health-economic inventory management model. This paper seeks to answer the following questions:

- How much of each corneal product and in which planning periods should be ordered?

- Which patient's demand should be met first to observe the health equity and the desired level of medical services on the one hand, and the least amount of shortage occurs on the other hand?

- How does the system deal with the emergency condition and demands with differentiated priorities to pose the least risk to the patients' health?

- How and in what order is the inventory issued and assigned to the demands while the least amount of wastage occurs?

The rest of this paper is organized as follows: Sect. 2 reviews the related literature. The applied methodology and the proposed mathematical model are described in Sects. 3 and 4, respectively. Section 5 investigates a real case study, including solutions, obtained results, and a discussion over the findings. Moreover, some sensitivity analyses are conducted over some of the critical parameters in this section. Finally, Sect. 6 presents the conclusion and future streams.

\section{Literature review}

The supply chain problems of perishable items, especially drugs and blood products, have been considered by many researchers since the 1960s (Pirabán et al. 2019; Samani et al. 2019a; Hosseini-Motlagh et al. 2020a). In this regard, a summary of the recently published papers on the related topics is provided in Table 1. Moreover, a structured review of the most related papers is conducted in this section. Finally, the research gap and the main contributions of the current study are investigated.

\subsection{Inventory management applications for healthcare supply chains}

The main focus of the HSC is to provide advanced healthcare services to patients (Manrodt and Schott 2016). While the cost of the service providing is increasing, one should effectively manage the healthcare systems to reduce the unnecessary costs of the system (Rajendran and Ravindran 2017; Attari and Abdoli 2020; Hosseini-Motlagh et al. 2020c). A review of the previous research shows that various mathematical methods have been employed to model healthcare systems' problems. In this regard, the HSC operations have been investigated to optimize the chain's inventory quantity upstream/downstream simultaneously (Franco and Alfonso-Lizarazo 2017). 


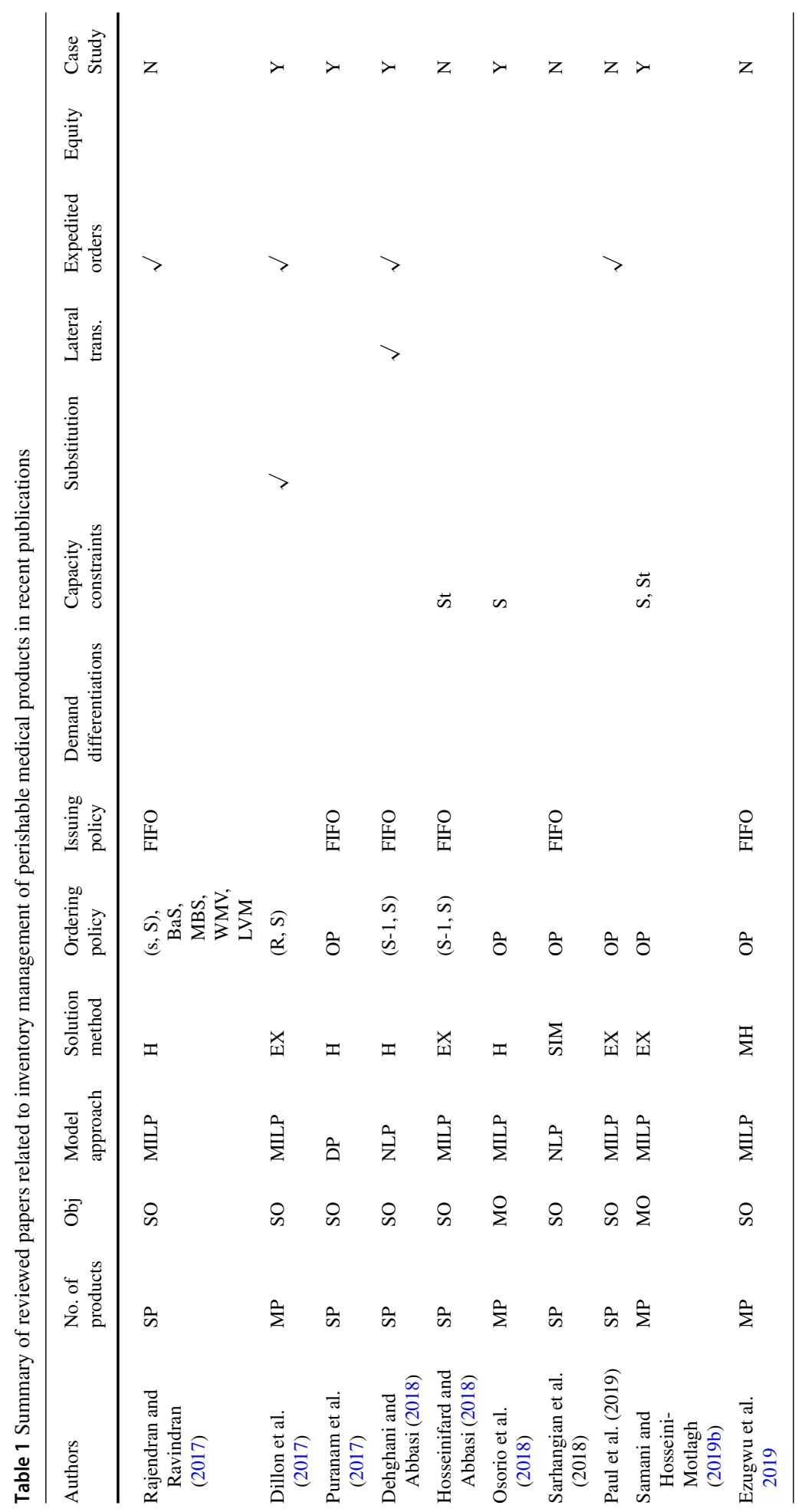

照 Springer $S \mathrm{D} / \mathrm{M}$ 


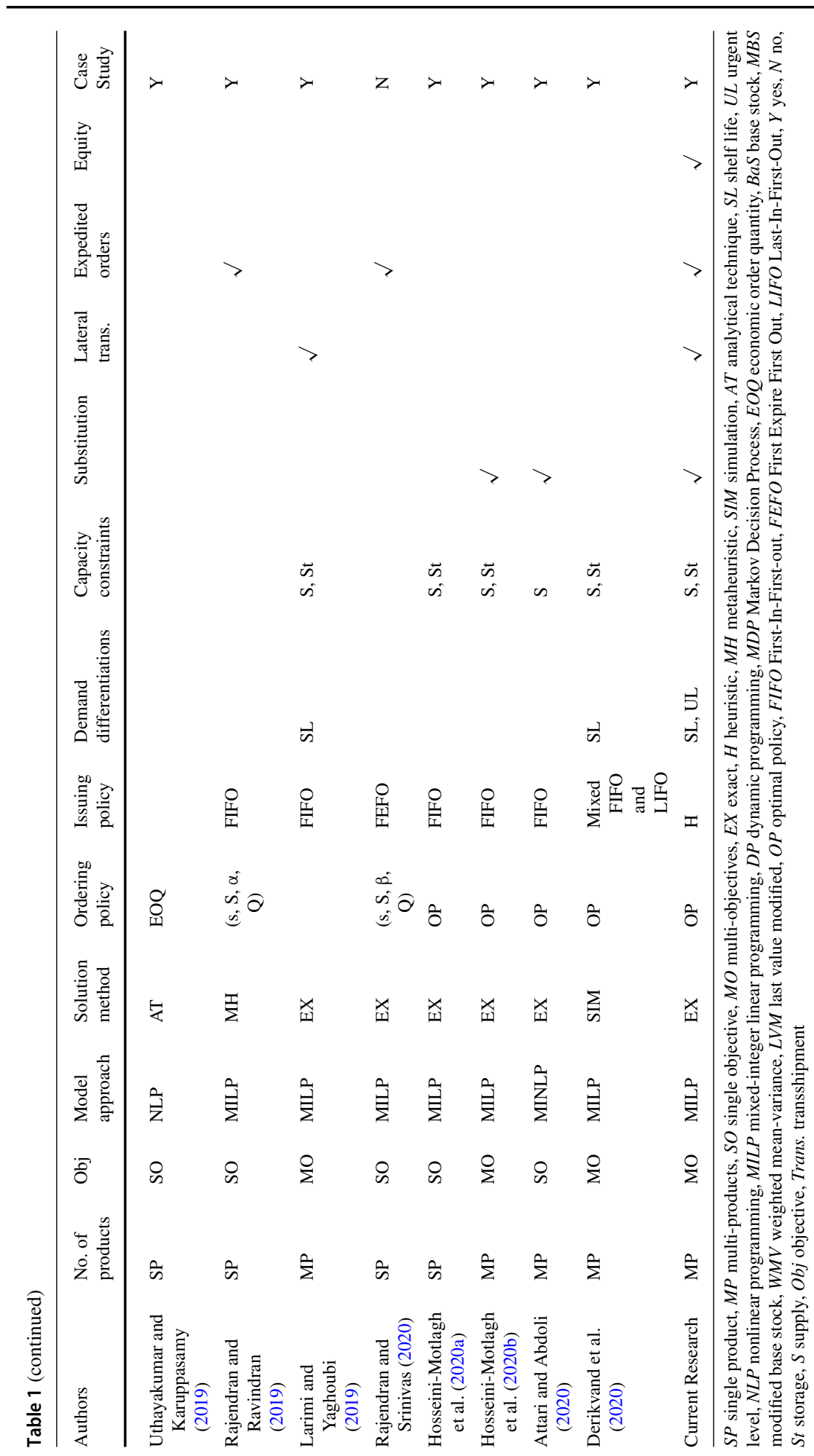

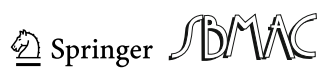


Most research in this field has focused mainly on minimizing inventory costs using various modeling tools and techniques to increase efficiency and effectiveness in the overall management of the HSC (Rajendran and Ravindran 2019). As Table 1 addresses, single or multiple objectives are considered in the literature. The modeling methods can be categorized into mathematical programming, simulation, and other methods. The mathematical programming methods mainly consist of mixed-integer linear programming (MILP) models and mixed-integer nonlinear programming (MINLP) models. Studies that developed mathematical modeling, in most cases, use exacts, heuristics, and metaheuristic solution approaches (Pirabán et al. 2019).

Most extended mathematical models in medicine inventory management have been conducted in a deterministic environment because of the complexity of coping with uncertainty (Hosseini-Motlagh et al. 2020c). However, more attention has been paid to supply chain's uncertainties in the last 2 decades (Mousazadeh et al. 2018).

The replenishment policies such as periodic review, continuous review, or modified policies are used to determine the optimal ordering quantity and replenishment periods (Dillon et al. 2017; Dehghani and Abbasi 2018; Derikvand et al. 2020; Rajendran and Srinivas 2020). Considering the lead-time as zero or constant is a logical and common approximation for modeling such healthcare systems (Rajendran and Ravindran 2017; Hosseinifard and Abbasi 2018).

The two issuance policies of first-in-first-out (FIFO) for the consumption of older products and last-in-first-out (LIFO) for fresher products had the highest application in the literature. Moreover, some studies have suggested modified hybrid and heuristic policies (Civelek et al. 2015; Rajendran and Ravindran 2017). Operationally, the items that first enter the storage do not necessarily have the maximum shelf life. Therefore, it is better to consider agebased issuance policies to manage perishable inventories. Ideally, it is better to consume older products earlier to avoid their wastage, but this is not always possible. It may change depending on medical preferences and patient conditions (Saha and Ray 2018). Nevertheless, in cases of shortage, substitution is a possibility based on medical priorities (Najafi et al. 2017; Sohrabi et al. 2021a).

Some of the previous studies considered the emergency as an instance of a shortage in the system. In practice, unsatisfied demand, especially emergencies, may be satisfied through an expedited order policy (Pirabán et al. 2019; Rajendran and Ravindran 2019; Derikvand et al. 2020). Note that this assumption technically has no difference from assuming "lost demand" in inventory management. The excess demand does not impact either the inventory composition or the usage in future periods. Although, a penalty cost is incurred for each unit of demand that cannot be satisfied immediately from on-hand inventory (Civelek et al. 2015).

Several papers are focused on managing the wastage of inventories with possible transshipment options. In this case, a lateral transshipment policy is considered (Hosseinifard and Abbasi 2018). Therefore, nearly outdated product units in the on-hand inventory may be transshipped to the supplier or carried over the next period. Note that the transshipment takes place among the hospital and the supplier and is only allowed for the units of the near-outdated product. There are agreements between hospitals and the supplier to transship nearly outdated units at a discount price; the demand is abundant and called a "clearance sale". Transshipment is not permissible for all blood units with age less than the transshipment threshold since the hospital must be prepared for non-elective demands and emergency conditions (Najafi et al. 2017).

The problem of inventory management of substances of human origin (SoHO) is fundamental, and recommending an effective and efficient solution will be very beneficial for the better functioning of any healthcare system. To the best of the authors' knowledge, the 
inventory management problems of the critical perishable products originated from human organ resources have received less attention so far by the researchers. An extensive body of research in this context includes blood products and has focused chiefly on minimizing the system costs, shortage, and wastage using different optimization techniques to increase the blood supply chain's (BSC's) efficiency and effectiveness (Habibi-Kouchaksaraei et al. 2018; Hosseini-Motlagh et al. 2020a).

\subsection{Health equity to access healthcare services}

Health equity is an essential criterion of services. Heretofore, many healthcare researchers have conceptually described health equity for medical services (Balarajan et al. 2011). Health equity entails that a patient is appropriately treated in various aspects of the disease for healthcare systems. This requirement is consistent with a familiar distinction between horizontal and vertical health equity (Culyer 2001). In healthcare systems, horizontal health equity requires similar medical services for similar patients. Vertical health equity entails dissimilar medical services for dissimilar patients in terms of the medical ULs, and commensurate with their illness (Regidor et al. 2008). The horizontal and vertical health equity is illustrated graphically by Fig. 1 . The review of the existing literature shows that almost all of the previous studies in this area were qualitative. This issue can be challenging because there are different ways to define equity, evaluate its effects, and classify patients to employ health equity. Therefore, a definition is needed to be operationalized based on measurable criteria (van Roode et al. 2020; Sohrabi et al. 2021a).

Introduction of the novel coronavirus disease has disrupted the supply of SoHO globally. It is because donors became anxious regarding the risk of the COVID-19 infection during the donation. Significant reduction in donations along with concerns regarding the safety of blood products has emerged. Therefore, service providers face the inevitable hazard of SoHO shortage (Al Mahmasani et al. 2021; Gupta et al. 2021). In this regard, health equity could be of utmost importance during the COVID-19 pandemic (Shamsoddin 2020). Due to the impact of the COVID-19 pandemic on SoHO, there is a need to adequately respond to ensure

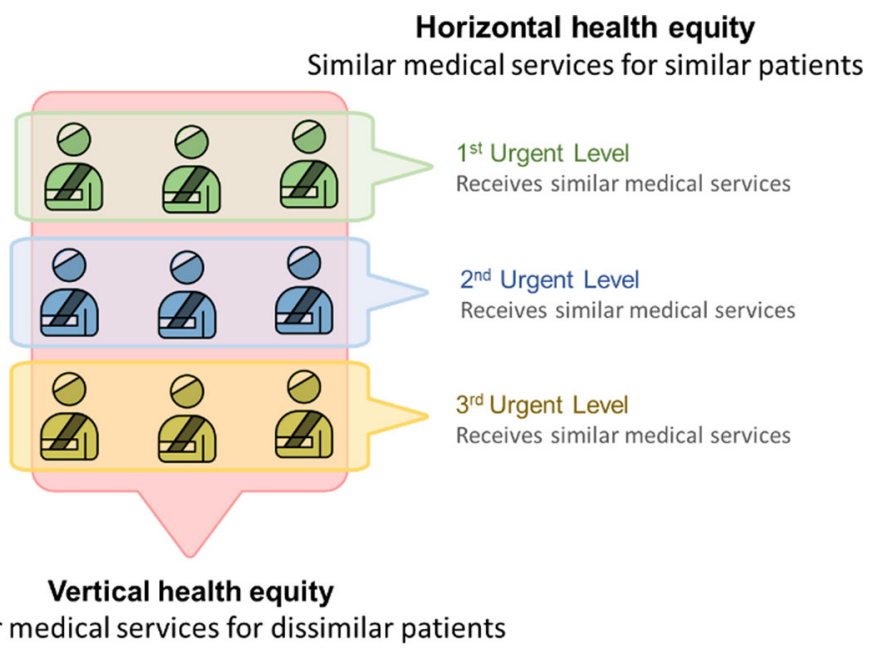

Fig. 1 Horizontal and vertical health equity 
the maintenance of core services (Assessment 2020). The pandemic has significantly stressed public health systems worldwide and exposed the gaps in healthcare for underserved and vulnerable populations. In the context of health equity, focusing on health system preparedness is paramount for protecting the health of all of society (Benjamin 2020).

Providing equity in health and participating in the acts of providing equitable healthcare have different aspects. Although this pandemic is not the first global-scale biological threat that humankind has faced, some points are still further addressed. Deeming health as a public good inevitably necessitates taking responsibility for its fair provision.

To the best of the authors' knowledge, few papers have focused on health equity implementation in operational research approaches to optimize HSC performance. In this regard, in one of the recent studies, the health equity in the BSC was considered for three types of injuries, which were differentiated in terms of medical ULs and demand satisfaction preferences consist of: major injuries which are emergency and needs immediate treatments, moderate injuries which are less urgent compare to major injuries but should be treated as soon as possible, and minor injuries which can be treated with delay (Cheraghi and HosseiniMotlagh 2018).

\subsection{Eye banking}

Corneal transplantation is one of the most common eye surgeries, considered the most abundant and successful allogeneic transplantation in the world (Zare et al. 2011). Recent developments in storage equipment used in eye banking and corrective changes in eye care and surgical removal techniques have significantly impacted donor tissue viability and increased the number of the processed corneal tissues (PCTs). Even though raising cultural sensitivity and community awareness and the widespread use of health promotion programs for organ donation have led to some countries' self-sufficiency in meeting the PCT demand (Gain et al. 2016).

In 2012, 184,756 corneal transplantation surgeries out of 283,530 corneal tissues were performed in 116 countries, processed in 742 eye banks. The United States of America, with $199 \times 16^{-10}$ per capita, has the most number of corneal surgeries, followed by Lebanon with $122 \times 16^{-6}$ per capita, and Canada with $117 \times 16^{-6}$ per capita. While the global required corneal is supplied from only 82 countries. The investigations show that about $53 \%$ of the worldwide population has no corneal transplantation access (Rosenwasser et al. 2017).

Therefore, limited supply and insufficient access to eye donors make systematic inventory management approaches more crucial for eye healthcare services. The PCTs are divided into five standard categories named 'Excellent', 'Very good', 'Good', 'Fair', and 'Poor', according to some qualitative characteristics such as corneal endothelial cells, cellular cross-section, and percentage of hexagonal cells.

The PCTs can usually be stored for between 7 and 14 days (Malhotra et al. 2018). The two 'Excellent' and 'Very good' quality donor corneas are usually used for penetrating keratoplasty. In contrast, 'Very good', 'Good' quality donor corneas are usually used for lamellar keratoplasty. 'Fair' and 'Poor' quality donor corneas are mostly used for research and lab examinations or disposed of. Depending on the attending physician's diagnosis, some quality degrees of the PCT can be substituted in different surgeries, according to medical priorities and risk factors for corneal graft failure and rejection (Garcin et al. 2019). 


\subsection{Research gap and contributions}

To the best of the authors' knowledge, except for the BSC, the other crucial medical products (i.e., SoHO) have been less investigated. Meanwhile, the study on optimizing the eye supply chain (ESC), as the most abundant transplantable tissue after blood, has been neglected. Although the research on whole-globe harvesting of the corneal tissue and its processing has been highly welcomed by eye health professionals in the last decade, ESC concerns have been neglected computationally using operational research techniques. The surveys show that most previous research has been carried out on eye-processing and corneal surgery techniques. In this respect, to the best of the authors' understanding, no study has been already conducted to present quantitative optimization models so far.

The increasing demand for eye products led to an increase in improving ESC management knowledge practically. Therefore, more attention needs to be paid to eye banking issues to provide optimal solutions for ESC problems. This important study adds further credibility to the importance of applying a practical approach for managing perishable medical inventories by developing a health-economic demand-oriented model for an entire healthcare system. Current research forms the cornerstone of future studies on mathematical optimization of transplantable organ tissues' supply chain problems, especially PCT. In summary, the main contribution of this survey in comparison with previous related works is as follows:

- Addressing the real-life concerns of corneal tissue inventory management systems quantitatively for the first time through utilizing operational research methods and applied mathematics;

- Implementing health equity pragmatically for corneal tissue inventory management to provide fair and equitable access to medical services for all patients' groups based on their medical priorities;

- Making a trade-off between the health equity and efficiency in the healthcare systems;

- Classifying demands and prioritize them based on blood age, substitution allowance, medical urgency, issuance and allocation policy, to ensure providing equitable services for all patients and reduce risk of life;

- Adopting a practical policy to face different demand classes considering emergencies;

- Implementing a hybrid empirical heuristic to issue and assign the blood inventory so that the slightest shortage and wastage occurs;

- Applying a real-life case to demonstrate the wide practicality of the proposed problem in healthcare systems.

\section{Methodology}

The proposed model in this research is a multi-objective mixed-integer linear programming (MOMILP) model. The objectives should be satisfied efficiently in a non-dominance space by finding global Pareto-optimal solutions. In the first step, a mathematical model is proposed. Then, a goal programming (GP) approach is employed to solve it in an actual situation. Next, the GP approach is described for making a trade-off among different objectives of the proposed model.

Different approaches have been proposed in the literature to solve the bi- or multi-objective decision-making (MODM) optimization problems. The GP approach is used when the number of goals is too much, while their importance is mostly the same (Gunantara 2018; Safaei et al. 2018; Zhang and Meng 2020). 
The methods applied for combining the objective functions into only one. GP is an old yet applicable solution method among available ones. This approach searches the feasible region and solution space of the optimization problem globally. GP is practical when the number of objectives is too much, while their importance is mostly the same (Gunantara 2018). It is selected to solve our multi-objective inventory management problem due to its close relation with linear programming. Minimizing shortage as well as inventory level, substitution, non-freshness, and total system costs are goals of this problem. Naturally, some goals may contradict others which their associate weights determine their importance. When decision-makers can comfortably determine each goal's weight, the GP approach seems appropriate (Mosadegh et al. 2017). That is why the GP approach is selected in this survey.

To implement the GP approach, a goal is first defined for every objective, ranging between 0 and 1 . The objective function tries to minimize the maximum deviation from each goal. Generally, a typical MODM problem is defined as follows (Jones and Tamiz 2016):

$$
\left\{\begin{array}{l}
\operatorname{Min}\left(f_{1}(x), f_{2}(x), \ldots, f_{n}(x)\right) \\
x \in X
\end{array} .\right.
$$

Given that the optimal value of each objective is shown by $f_{i}^{*}$, where $i=1,2, \ldots, n$. In real-world MODM problems, taking existing conflict into account among objectives, there is not usually a solution like $x^{*} \in X$., for which all objectives are optimized. Accordingly, if $A$ is a solution method and $x^{A}$ is its output, then, this solution approach is more efficient when $f_{i}\left(x^{A}\right)$ has less distance to $f_{i}^{*}$.

In the GP approach, an acceptable value is first considered for every objective (e.g., its optimal value, $f_{i}^{*}$ ). The goal function is then defined so that any reduction in the objective function linearly increases the goal value. Supposed that $m_{i}=f_{i}^{*}$ is considered as the minimum/ideal value of $i$ th objective and $M_{i}$ is defined as its upper bound. Therefore, in the GP method, the $i$ th objective could be defined as follows:

$$
G_{i}(x)=\mu(x)_{\mid f_{i}}=G\left(f_{i}(x)\right)= \begin{cases}0 ; & f_{i}(x) \geq M \\ \frac{M_{i}-f_{i}(x)}{M_{i}-m_{i}} ; & x \in X . \\ 1 ; & f_{i}(x) \leq m_{i}\end{cases}
$$

Then, using the GP method, MODM problems could be stated in terms of a single-objective model, as follows:

$$
\left\{\begin{array}{l}
\max z=\min \left\{G_{i}(x): \quad i=1: n\right\} \\
G_{i}(x)=\frac{M_{i}-f_{i}(x)}{M_{i}-m_{i}} ; \quad i=1,2, \ldots, n \\
x \in X
\end{array}\right.
$$

where if $f_{i}(x) \rightarrow m_{i}$, then, $G_{i}(x) \rightarrow 1$.

In some cases, instead of the formulation mentioned above, the minimax technique is used, which is employed in this research. In the minimax method, the maximum deviation from the optimal value of the objectives is minimized. The formulation of the minimax method 
could be stated as follows:

$$
\left\{\begin{array}{l}
\mathrm{GP} \\
\min L_{\infty}\left(d_{i}(x), i=1,2, \ldots, n\right) \\
d_{i}(x)=\frac{f_{i}(x)-m_{i}}{M_{i}-m_{i}} ; i=1,2, \ldots, n \\
x \in X
\end{array}\right.
$$

where $L_{\infty}$ signifies the extreme norm and $d_{i}(x)=\frac{f_{i}(x)-m_{i}}{M_{i}-m_{i}}$ is the relative deviation of each objective function from its optimal value. The formulation mentioned above could be linearized as follows:

$$
\left\{\begin{array}{l}
\mathrm{GP} \\
\min Z \\
Z \geq \frac{f_{i}(x)-m_{i}}{M_{i}-m_{i}} ; i=1,2, \ldots, n \\
x \in X
\end{array}\right.
$$

\section{Problem statement and mathematical modeling}

The design of the current study is conducted according to real-world concerns. In this research, the shown network in Fig. 2 is investigated from a medical center's perspective for managing the inventory of perishable products. The ideal is to obtain inventory management plans for the different demand classes appropriately. In this regard, the health and economic objectives are to be satisfied, i.e., equity-oriented treatment (health objectives) and cost minimization (economic objective), without any performance reduction in health objectives. In this study, to obtain health equity, three objectives are considered. First, the total shortage minimization;

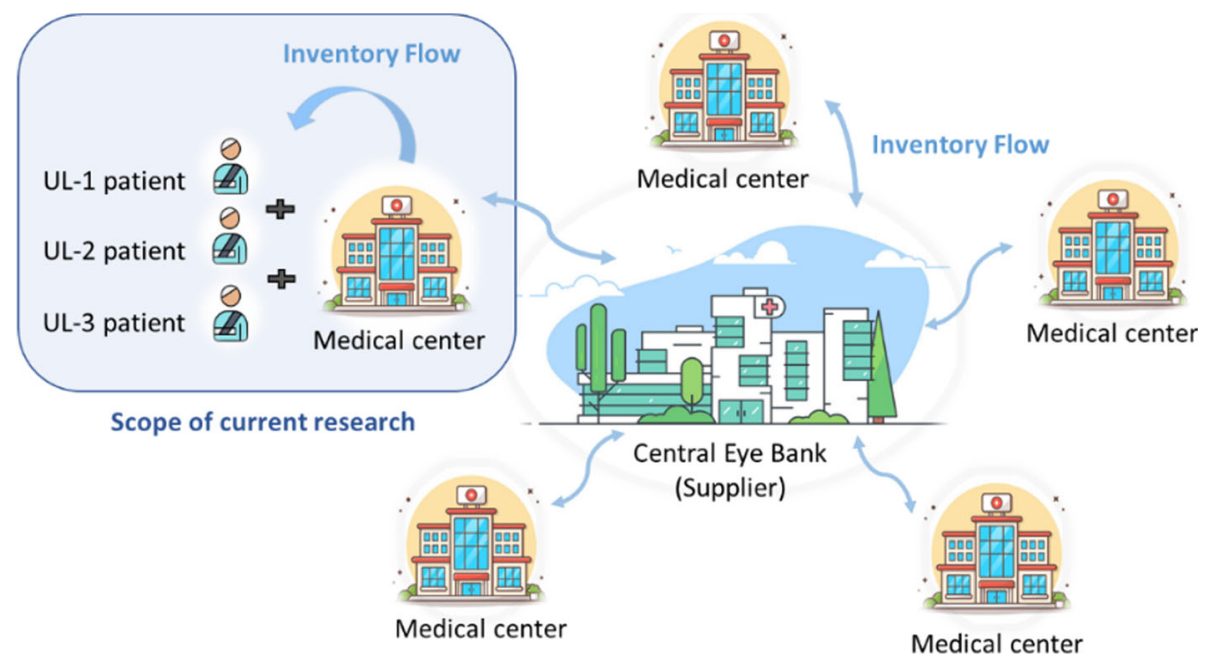

Fig. 2 Proposed system 
it should be noted that the shortage may only occur in the third level of demand priority (low priority demand) and has a specific value for each product. Second, substitution minimization of a particular demand satisfaction with other substitutable products based on medical priorities (some products with a given substitution rate could be replaced). Third, minimization of demand satisfaction with a non-fresh product to reduce immunological and infection risks. The freshness for each product and each demand class has a specific value.

\subsection{Problem statement}

A specific medical product can be prescribed to treat diseases in different urgent classes. Accordingly, healthcare systems mostly deal with health equity concerns in providing services with the least health risks. The current investigation seeks to find optimal ways to meet the PCT demands health-economically, with minimal shortage and wastage for the real-world medical systems. Generally, and in the current research, patients' demands are separated into three priority classes considering health equity, named 'High', 'Medium', and 'Low', according to the patient medical condition and treatment urgency (Sohrabi et al. 2021a; b).

In the current study, the product items in their first half-life are considered fresh. Those product items that have passed their half-life are considered regular in freshness, and at the end of their shelf life, they are expired and will be discarded. The discrete demands are periodically (daily), distinct and independent. The patient's UL, substitution allowance, and freshness value are considered for the differentiated demand classes, separately. The patient-oriented treatment causes the priority of demand satisfaction not to be the same at the differentiated demands class. Some optimal hybrid policies are presented to satisfy the demand at different ULs and reduce product shortage and wastage.

Suppose the demand for a given product cannot be satisfied with the same product type. In that case, one can consider a shortage-based substitution policy, according to the patient's UL, or use expedited service for an immediate replenishment. However, the expedited orders occasionally happen in real-world cases where there are severe risks to the patient's health. In the case of using expedited service, an extra cost is imposed on the system. This kind of shortage can hurt the organization's reputation, so more optimization must be done to prepare the system to encounter emergency conditions (Derikvand et al. 2020). The penalty cost is mainly related to utilizing an immediate transportation service and restricting expedited orders (Civelek et al. 2015). It should be mentioned that there are always some safety stocks in the eye bank storage for emergency conditions. It is not offered to use expedited service as much as possible. After adopting the extended assignment and issuance hybrid policies, if some parts of the demands have not yet been satisfied, they would be lost.

Due to the human organ transplants' criticality and finite resources, the PCT's wastage and expiration are not acceptable for the HSC. Accordingly, medical centers that request PCT to treat the patients try to prevent its wastage to the optimal extent due to the relative social and economic costs for the HSC. In general, the eye care instructions emphasize transferring the donated tissue from the supply node to the demand node only once to minimize the risk factors for corneal graft failure and rejection (Panda et al. 2007; Williams and Coster 2007). Nevertheless, in rare real-world cases, where the transplantable tissue is expiring, it can be transferred to the eye bank to be used to save another patient's health. Therefore, to prevent wastage, for nearing expiration items, a lateral transshipment threshold is considered. Suppose there is no demand for an available product unit by arrival transshipment threshold. In that case, it will be transferred to the supplier (for other medical centers' demand satisfaction) by receiving a part of the unit purchasing price, the so-called auction price. 


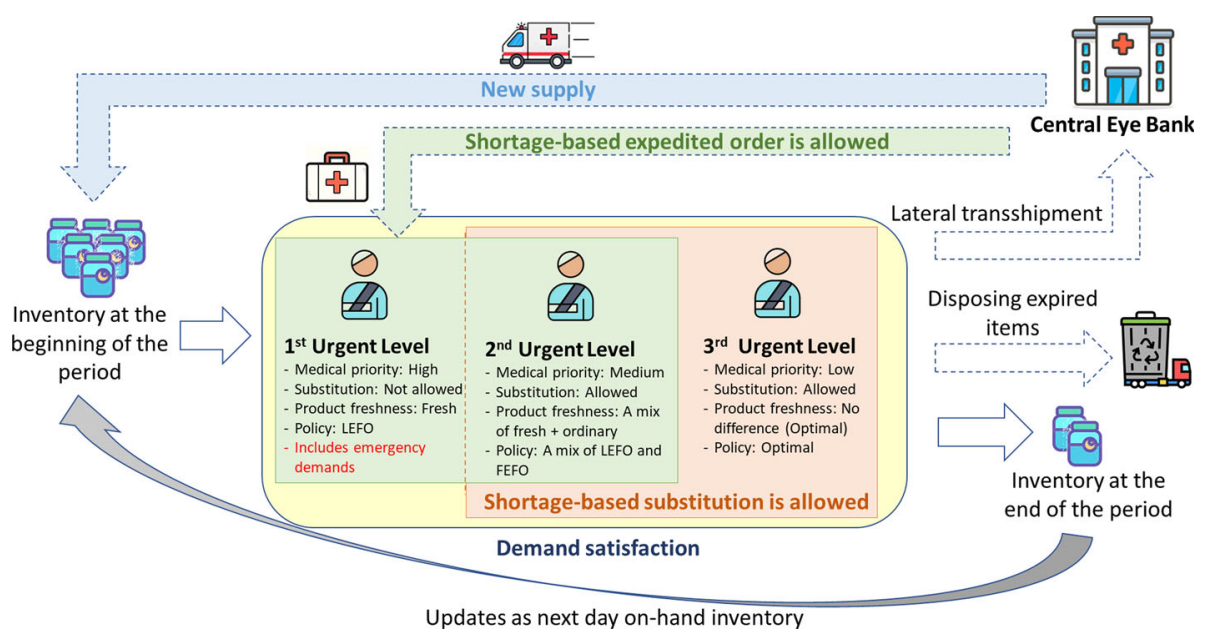

Fig. 3 The inventory flows and demand classification in the proposed system

The expired items impose a wastage cost on the system. Wastage cost is an intrinsic value of product and disposal cost (Najafi et al. 2017). Biological wastages must be salvaged by incineration (Evans 2014; Rajendran and Ravindran 2019). This policy has been developed to minimize the risk of infection exposure and the environmental side effects. Hence, the model must account for the wastage (or outdating) cost. These costs include the cost of retrieving, transferring, and autoclaving the biohazard waste. It is taken as the purchasing cost based on the ratios given in the literature (Rajendran and Ravindran 2017, 2019).

The supply is capacitated and carried out only by one leading supplier. In addition, the products received from the supplier have a specific shelf life. The demands are periodic (daily) and independent. Figure 3 indicates the inventory flow in the proposed system. The following is presented on how to meet the demand for different levels of urgency:

- Demand for tertiary care (UL-1) The highest priority demand is only for the freshest items due to avoiding medical risk. The same item could only meet the product demand at this level, so the substitution in this UL is not allowed. It would be lost and ordered immediately by imposing an expedited service penalty cost in case of shortage. An appropriate issuance policy, so-called the last-expire-first-out (LEFO), is considered for this UL to assign the freshest items to the demand.

- Demand for secondary care (UL-2) The moderate priority demand has lower medical sensitivity compared to the UL-1, but there is still some medical risk; therefore, similar to UL-1, this level belongs to the fresh items but follows a different pattern. The issuance policy for satisfying UL-2 demand is a mix of the first-expire-first-out (FEFO) and LEFO policies. In this case, the demand is first met with those in their first half-life (which is considered fresh); however, it has the least remaining shelf life. In this case, the FEFO issuance policy is used for demand satisfaction. According to the medical priorities, shortage-based substitution with the freshest items of other types of products is allowed at this level and follows the LEFO issuance policy. If there is still some unmet demand after the substitution, it would be lost and ordered immediately through imposing expedited service penalty costs. 
- Demand for primary care (UL-3) The lowest priority demand encounters the least level of health risk and medical sensitivity. Therefore, the products' remaining shelf life is not that important for demand satisfaction; however, it is preferred to use the fresher ones to treat patients. Suppose some parts of demands could not be met. In that case, shortage-based substitution with other types of products is allowed, according to the medical priorities. If some demand parts are still unsatisfied, the shortage would be lost and would not be ordered through expedited services. The issuance policy is considered for satisfying the demand at UL-3 is the FEFO issuance policy starting with the oldest-age units.

\subsubsection{Assumptions}

The following assumptions are considered to conduct the current research:

- The planning horizon is finite, including some specific periods (e.g., 1-month plan with daily periods).

- The supplier capacity is limited and variable in each period.

- The demand for each product varies in each period.

- The shelf life of all products is the same.

- The products are received in the freshest form from the supplier.

- The medical system's storage capacity is limited, and a maximum holding capacity is already determined for each product.

- The allowed ordering periods are already specified and are a subset of the planning horizon periods.

- The demand is considered in three classes, UL-1, UL-2, and UL-3, where the UL-1 has the highest medical priority, then UL-2, and finally, UL-3 with the lowest medical priority.

- The demand back-order is not allowed, and each period's demand must be satisfied in the same period, not the next.

- The shortage is allowed only for the demand with UL-3. In case of a shortage of demands with UL-1 and UL-2, one should use expedited services, implying a high penalty cost to the system.

- The importance of product availability for each UL's patients is determined by medical experts, ranging between 5 and 10 (the number 10 is the most important).

- The substitution rate of products ranges between 0 and 1 , specified by medical experts; 1 signifies fully compatible, and 0 means non-compatible.

- Each product's freshness importance in each UL ranges between 5 and 10, specified by medical experts (the number 10 is the most important).

- The products at their first half-life are considered fresh, and in their second half-life, they are considered non-fresh (regular).

- Lead time to receive the order from the supplier is deterministic (one period). In addition, expedited orders are received immediately in the same period from the supplier.

- The initial inventory of the system is negligible.

\subsubsection{Notations}

The notations and symbols used in the proposed mathematical model are as follows. The mathematical formulation is presented in Table 2. 
Table 2 List of indices, parameters, and decision variables of the proposed model

Sets and indices

$T$ : Set of planning periods (day), $T=\{1,2, \ldots, t, \ldots,|T|\}$

$R$ : Allowed replenishment periods (day), $R \subseteq T$

$P$ : Set of product types, $P=\{1,2, \ldots, p, \ldots,|P|\}$

$U$ : Set of demand priorities (demand classes), $U=\{1,2, \ldots, u, \ldots,|U|\}$

$S^{p}$ : Set of substitutable products, $S^{p} \subseteq P$

$I$ : Set of products shelf life (day), $I=\{1,2, \ldots, i, \ldots,|I|\}$

Parameters

$o c$ : Fixed-cost of purchasing products (currency/unit)

$p c_{p t}$ : The variable purchasing cost of product $p$ at period $t$ (currency/unit)

$b c_{p t}$ : Expedited service cost of product $p$ at period $t$ (currency/unit)

$h_{p i}$ : Inventory holding cost of product $p$ with age $i$ (currency/unit)

$e_{p}$ : Cost of expired/outdated products (currency/unit)

$\theta_{p i}$ : The auction price of nearing expiration product $p$ and age $i$ (currency/unit)

$M A_{p}$ : Maximum number of nearing expiration product $p$ which can be transshipped to the supplier at each period (unit)

$\operatorname{dem}_{p u t}$ : Demand for product $p$ with priority $u$ at period $t$ (unit)

$\operatorname{cap}_{p t}^{\text {sup }}$ : Maximum supplier capacity to provide product $p$ at period $t$ (unit)

Vol: Total storage capacity of the medical system (unit)

$\gamma_{p}$ : The storage capacity of the medical center for one unit of product $p$ (unit)

$m v_{p}$ : The maximum storage capacity of the medical system for product $p$ (unit)

$\alpha_{p u}$ : The relative importance of the availability of product $p$ for patients with priority $u$

$\beta_{p u}$ : The relative importance of freshness of product $p$ for patients with priority $u$

$\Delta_{p p^{\prime} u}$ : Substitution rate of product $p$ with product $p^{\prime}$ for demand with priority $u$

$\operatorname{Tr}$ : Transshipment threshold (day)

$\phi_{p u}: 1$ if it is possible for demand with priority $u$ to substitute product $p$ with another product, otherwise 0

$L T$ : Lead time for replenishment delivery

Decision variables and outputs

$Q_{p t}$ : The order quantity for product $p$ at period $t$ (unit)

$I_{p i t}$ : Inventory level of product $p$ with age $i$ at period $t$ (unit)

$Y_{\text {pit }}$ : Amount of assigned/consumed inventories of product $p$ with age $i$ for total demands satisfaction at period $t$ (unit)

$F_{\text {piut }}$ : Amount of assigned/consumed inventories of product $p$ with age $i$ for satisfying demand with priority $u$ at period $t$ (unit)

$F_{\text {pip } p^{\prime} u t}^{\prime}$ : Amount of substitute inventories of product $p$ with age $i$ for satisfying demand of product $p^{\prime}$ with priority $u$ at period $t$ (unit)

$D_{p u t}$ : Total satisfied the demand for product $p$ with priority $u$ ar period $t$ (unit)

$B_{\text {put }}$ : Total unfulfilled demand of product $p$ with priority $u$ at period $t$, before expedited orders (unit) 
Table 2 (continued)

\section{Decision variables and outputs}

$W_{\text {put }}$ : Amount of expedited orders for product $p$ for demand with priority $u$ at period $t$ (unit)

$A_{\text {pit }}$ : Amount of transshipped units of product $p$ with ag $i$ at period $t$ (unit)

$E_{p t}$ : Amount of Expiration of product $p$ at period $t$ (unit)

$O_{t}$ : Binary variable; 1 if the system replenishes any product units at period $t$; Otherwise 0

$Z^{E 1}$ : Value of economic objective function (total cost)

$Z^{S 1}$ : Value of first health objective function (shortage)

$Z^{S 2}$ : Value of second health objective function (substitution)

$Z^{S 3}$ : Value of third health objective function (non-freshness)

\subsection{The objective functions}

The current research comprises four objective functions, where one of which deals with economic issues. The rest of the other three ones are concerned with healthcare social matters. Here, it is presented how to model each of the objectives.

\subsubsection{Economic objective}

The economic objective minimizes the total costs of inventory management. This function comprises five main parts: in the first part, the fixed and variable costs of the products and purchasing costs are calculated. In the second part, the purchasing cost of products using expedited service is computed to compensate for the shortage. In the third part, the holding cost is calculated, and in the fourth part, the wastage/disposal cost of expired products is computed. Finally, the fifth part computes the obtained auction revenue of near-expiration products. The following equation shows the economic objective function and its different parts:

$$
\begin{aligned}
\min Z^{E 1}= & {\left[\sum_{t} o c \times O_{t}+\sum_{p} \sum_{t} p c_{p t} \times Q_{p t}\right]+\left[\sum_{p} \sum_{t} b c_{p t} \times W_{p t}\right] } \\
& +\left[\sum_{p} \sum_{i} \sum_{t} h_{p i} \times I_{p i t}\right]+\left[\sum_{p} \sum_{t} e_{p} \times E_{p t}\right] \\
& -\left[\sum_{p} \sum_{i \geq T r=\frac{3|I|}{4}} \sum_{t} \theta_{p i} \times A_{p i t}\right] .
\end{aligned}
$$

\subsubsection{The first health objective}

The first health objective minimizes the inventory shortage. The shortage of medical products in healthcare systems has a direct impact on patients' health. Equation (7) indicates how the first health objective function is calculated, by which the shortage is minimized. In other words, Eq. (7) minimizes the unsatisfied demand after utilizing normal and expedited ordering 
policies. $\alpha_{p u}$ is a coefficient to show the importance of existing product $p$ in the system. The higher the value of $\alpha_{p u}$ for a given product is, the less the shortage amount for that product, and therefore, the less the shortage objective function value:

$$
\min Z^{S 1}=\sum_{p} \sum_{u} \sum_{t} \alpha_{p u}\left(B_{p u t}-W_{p u t}\right) .
$$

Note that the value of different products for different ULs is considered to be stated in a standard manner. In addition, the lost shortage may only happen in UL-3 for the current problem.

\subsubsection{The second health objective}

The second health objective deals with minimizing the shortage-based substitution of products in demand with the rest of available products. The following equation shows the second health objective, by which the number of substitutions (specifically inappropriate substitution) is minimized:

$$
\min Z^{S 2}=\sum_{p} \sum_{i} \sum_{p^{\prime} \in S^{p}} \sum_{u} \sum_{t}\left(1-\Delta_{p p^{\prime} u}\right) F_{p i p^{\prime} u t}^{\prime} .
$$

Note that different products can be substituted with a specific substitution rate. Therefore, the less the substitution rate is, the more the second objective function's sensitivity concerning substitution, which results in fewer total substitutions.

\subsubsection{The third health objective}

The third health objective is concerned with the freshness rate of items units assigned for demand satisfaction. This objective function is shown in Eq. (4), in which demand satisfaction with non-fresh products is minimized. As already mentioned, the freshness for each product and each demand priority has a specific value, where a product is considered as non-fresh, if its age is more than $\frac{|I|}{2}$. In addition, the relative importance coefficient of the freshness is multiplied by the degree of the non-freshness $\left(\frac{\left(i-\frac{|I|}{2}\right)}{|I|}\right)$ to increase the sensitivity to the non-fresh units:

$$
\begin{aligned}
\min Z^{S 3}= & \left(\sum_{p} \sum_{i>\frac{|I|}{2}} \sum_{u} \sum_{t} \frac{\beta_{p u}\left(i-\frac{|I|}{2}\right)}{|I|} F_{p i u t}\right. \\
& \left.+\sum_{p} \sum_{i} \sum_{p^{\prime} \in S^{p}} \sum_{u} \sum_{t} \frac{\beta_{p u}\left(i-\frac{|I|}{2}\right)}{|I|} F_{p^{\prime} p^{\prime} u t}^{\prime}\right) .
\end{aligned}
$$

Four health-economic objectives are considered, including one economic and three health objectives, dealing with health equity in healthcare. In classic approaches, mostly shortage is taken as a shortage cost into account in the economic objective, but due to the importance of shortage in this research, this objective is considered independently. 


\subsection{Constraints}

$$
\begin{aligned}
& Q_{p t} \leq \operatorname{cap}_{p t}^{\sup _{t}} . O_{t} ; \forall p \in P, t \in T \\
& O_{t}=0 ; \forall t \notin R \\
& Q_{p(t-L T)}=Y_{p 1 t}+I_{p 1 t} ; \forall p \in P, t \in T \\
& I_{p(i-1) t-1}=Y_{p i t}+I_{p i t}+A_{p i t} ; \forall p \in P, i \in I-\{1\}, t \in T \\
& \left\{\begin{array}{c}
A_{p i t}=0 ; \forall p \in P, i \leq\left(|I|-T r=\frac{3|I|}{4}\right), t \in T \\
A_{\text {pit }} \leq M A_{p} ; \forall p \in P, i>\left(|I|-T r=\frac{3|I|}{4}\right), t \in T
\end{array}\right. \\
& Y_{\text {pit }}=\sum_{u} F_{\text {piut }}+\sum_{p^{\prime} \in S^{p p^{\prime}}} \sum_{u} F_{\text {pip } p^{\prime} u t}^{\prime} ; \forall p \in P, i \in I, t \in T \\
& D_{\text {put }}=\text { dem }_{\text {put }}-B_{\text {put }} ; \forall p \in P, u \in U, t \in T \\
& \sum_{i} F_{p i u t}+\sum_{p^{\prime} \in S^{p p^{\prime}}} \sum_{i} F_{p^{\prime} i p u t}^{\prime}=D_{p u t} ; \forall p \in P, u \in U, t \in T \\
& W_{\text {put }}=B_{\text {put }} ; \forall p \in P, u \in U-\{3\}, t \in T \\
& W_{p 3 t} \leq B_{p 3 t} ; \forall p \in P, t \in T \\
& E_{p t}=I_{p|I| t-1} ; \forall p \in P t \in T \\
& \sum_{p} \sum_{i} \gamma_{p} . I_{p i t} \leq v o l ; \forall t \in T \\
& \sum_{i} \gamma_{p} . I_{p i t} \leq m v_{p} ; \forall p \in P, t \in T \\
& B_{p 1 t}>0 \Rightarrow \sum_{u>1} F_{p i u t}+\sum_{p^{\prime} \in S^{p}} \sum_{u>1} F_{p i p^{\prime} u t}^{\prime}=0 ; \forall p \in P, i \in I, t \in T \\
& B_{p 2 t}>0 \Rightarrow \sum_{u>2} F_{p i u t}+\sum_{p^{\prime} \in S^{p}} \sum_{u>2} F_{p i p^{\prime} u t}^{\prime}=0 ; \forall p \in P, i \in I, t \in T \\
& \sum_{i} F_{p i p^{\prime} u t}^{\prime} \leq \phi_{p u} M ; \forall p \in P, p^{\prime} \in S^{p}, u \in U, t \in T \\
& I_{p i t}>0 \Rightarrow \sum_{j>i} F_{p j u t}=0 ; \forall p \in P, i \in I, t \in T,[u=1] \\
& \left\{\begin{array}{l}
\ell_{p i t} \geq \frac{I_{p i t}}{M} ; \forall p \in P, i \in I, t \in T \\
\ell_{p i t} \leq 1+\frac{I_{p i t}-e p s}{M} ; \forall p \in P, i \in I, t \in T \\
\sum_{j>i} F_{p j u t} \leq\left(1-\ell_{p i t}\right) M ; \forall p \in P, i \in I, t \in T,[u=1] \\
\ell_{p i t} \in\{0,1\}
\end{array}\right. \\
& I_{\text {pit }}>0 \Rightarrow \sum_{j<i} F_{p j u t}=0 ; \forall p \in P, i \leq \frac{|I|}{2}, t \in T,[u=2]
\end{aligned}
$$




$$
\begin{aligned}
& I_{p i t}>0 \Rightarrow \sum_{j>i} F_{p j u t}=0 ; \forall p \in P, i>\frac{|I|}{2}, t \in T,[u=2] \\
& \left\{\begin{array}{l}
\ell_{p i t} \geq \frac{I_{\text {pit }}}{M} ; \forall p \in P, i \in I, t \in T \\
\ell_{\text {pit }} \leq 1+\frac{I_{\text {pit }}-e p s}{M} ; \forall p \in P, i \in I, t \in T \\
\sum_{j<i} F_{p j u t} \leq\left(1-\ell_{\text {pit }}\right) M ; \forall p \in P, i \leq \frac{|I|}{2}, t \in T,[u=2] \\
\ell_{\text {pit }} \in\{0,1\}
\end{array}\right. \\
& \left\{\begin{array}{l}
\ell_{\text {pit }} \geq \frac{I_{\text {pit }}}{M} ; \forall p \in P, i \in I, t \in T \\
\ell_{\text {pit }} \leq 1+\frac{I_{\text {pit }}-e p s}{M} ; \forall p \in P, i \in I, t \in T \\
\sum_{j>i} F_{p j u t} \leq\left(1-\ell_{p i t}\right) M ; \forall p \in P, i>\frac{|I|}{2}, t \in T,[u=2] \\
\ell_{\text {pit }} \in\{0,1\}
\end{array}\right. \\
& I_{p i t}>0 \Rightarrow \sum_{j<i} F_{p j u t}=0 ; \forall p \in P, i \in I, t \in T,[u=3] \\
& \left\{\begin{array}{l}
\ell_{p i t} \geq \frac{I_{p i t}}{M} ; \forall p \in P, i \in I, t \in T \\
\ell_{p i t} \leq 1+\frac{I_{p i t}-e p s}{M} ; \forall p \in P, i \in I, t \in T \\
\sum_{j<i} F_{p j u t} \leq\left(1-\ell_{p i t}\right) M ; \forall p \in P, i \in I, t \in T,[u=3] \\
\ell_{p i t} \in\{0,1\}
\end{array}\right. \\
& \left\{\begin{array}{l}
O_{t}, \ell_{p i t} \in\{0,1\} \\
Q_{p t}, I_{p i t}, Y_{p i t}, F_{p i u t}, F_{p i p^{\prime} u t}^{\prime}, G_{p u t}, D_{p u t}, B_{p u t}, W_{p u t}, A_{p i t}, E_{p t} \geq 0 ; G_{p u t} \leq 1
\end{array}\right.
\end{aligned}
$$

Equation (10) shows the limited supply capacity and states the order quantity can be equal to or less than the supply capacity. Equation (11) ensures that the replenishment for PCT can only occur during allowed review periods. It should be mentioned that if $R=T$, this constraint is not necessary. Constraint (12) expresses that the total order quantity of the fresh units at period $t-1$ equals assigned units for demand satisfaction plus stock-on-hand at the end of period $t$. As mentioned previously, it is assumed that the products received by the medical center at the beginning of day $t$ are fresh, and they are in the first period of their shelf life. Note that the lead-time to receive the order quantity from the supplier is considered 1 day. Constraint (13) shows that the stock-on-hand of a given item with remaining shelf life of $i-1$ days at the end of period $t-1$ equals to the summation of the assigned units for demand satisfaction, transshipped units, and stock-on-hand with shelf life of $i$ days at the end of period $t$ of the planning horizon.

Constraint (14) first restricts a product unit's transshipment before the threshold to be kept as safety stock; second, there is an upper bound on each product's transshipment at each period. Constraint (15) indicates product $p$ assignment and substitution to meet $u$ th UL demand at day $i$ of their shelf life during period $t$ of the planning horizon. Equation (16) calculates the total satisfied demand of product $p$ at $u$ th UL and period $t$ of the planning 
horizon. In addition, the unfulfilled demand at $u$ th UL is considered as the shortage, namely $B_{\text {put }}$, at period $t$ of the planning horizon, which might be compensated by shortage-based substitution, ordered through expedited service, or remained as lost depending on the predetermined demand's related policies. Equation (17) shows how to satisfy the $u$ th UL demand for product $p$ at period $t$ of the planning horizon. In other words, Eq. 17 indicates how $u$ th UL demand for product $p$ is met by the same requested product or with other substitutable products if allowed.

Constraint (18) expresses that the demand for product $p$ at $u=1,2$ must be satisfied. In case of shortage, an extra order must be taken through expedited service. However, according to Eq. (19), it is unnecessary to compensate for the unfulfilled demand at $u=3$. Of course, it should be pointed out that the first health objective function tends to compensate unfulfilled at $u=3$ by ordering through expedited service. Equation (20) calculates the number of expired units at the period $t$ of the planning horizon. Accordingly, the inventory of any unit at the end of period $t-1$, which is at the last period of its shelf life, is considered as the expired unit at period t of the planning horizon. Constraints (21) and (22) address the total storage capacity of the system and the storage capacity limitation of each product at a period $t$ of the planning horizon.

Equation (23) guarantees that the demand at $u=1$ must be satisfied, where, as long as there is a shortage for demand at $u=1$, it is not possible to meet the demand of other ULs. Similarly, Eq. (24) guarantees the priority of $u=2$ over $u=3$. It also should be mentioned that if $\alpha_{p 1}>\alpha_{p 2}>\alpha_{p 3}$, the second objective function satisfies constraints (23) and (24) in optimality. Equation (25) shows how product substitution based on medical priorities can be performed at any UL For instance, if it is not possible to substitute any product at $u=1$, then, $\sum_{i} F_{\text {pip }{ }^{\prime} u t}^{\prime}=0$. It should be mentioned that $M$ is a very big number. Equation (26) addresses the LEFO issuance policy for demand satisfaction at $u=1$; In this way, the product with a shelf life of $j>i$ days should not be used to satisfy the demand until the quantity of the product inventory with a shelf life of $i$ days is exhausted. Equation (27) shows constraint (26) in a linear form. It should be mentioned that in Eq. (27), $l_{\text {pit }}$ is a binary variable, which would be 1, if $I_{\text {pit }}>0$. In constraint (26), $M$ and eps are, respectively, a positive big enough number and a very small number.

Equations (28) and (29) address how FEFO and LEFO issuance policies are applied to satisfying the demand at $u=2$. Equation (28) indicates that the fresh product units (with a shelf life of fewer than $I / 2$ days) must be issued based on FEFO policy; in this way, the product units with a shelf life of $j<i$ days should not be used for demand satisfaction until the inventory with a shelf life of $i$ days is exhausted. In addition, constraint (29) indicates that the fresh product units (with a shelf life of more than $I / 2$ days) must be issued based on LEFO policy; In this way, the product units with a shelf life of $j>i$ days should not be used for demand satisfaction, until the inventory with a shelf life of $i$ days is exhausted. The constraints (30) and (31) show Eqs. (28) and (29) in a linear form, respectively. Similarly, in constraints (30) and (31), $l_{\text {pit }}$ is a binary variable, which would be 1 , if $I_{p i t}>0$. In these constraints, $M$ and eps are, respectively, positive big enough and very small numbers.

As already pointed out, to meet the demand of patients for products at $u=3$ based on optimal policy (OP), the model itself decides which part of the inventory at the storage should be issued. But, if the FEFO issuance policy is considered for demand assignment at $u=3$, constraint (32) would be applied, where the production unit with a shelf life of $j<i$ days should not be used for the demand satisfaction until the product inventory with a shelf life of $i$ days is exhausted. The constraint (33) shows constraint (32) in a linear form. Constraint (34) represents non-negativity and binary restrictions in the model. 


\subsection{Solution approach}

To solve the problem using the goal programming approach defined in Sect. 3, the payoff matrix between health-economic objectives should be obtained in the beginning. In this regard, the proposed model is first solved four times, each of which comprises only one of the considered objectives in terms of a single-objective problem. To find the appropriate interval corresponding to the $i$ th objective $(i=2, \ldots, n)$, the following optimization problem should be solved for each objective, $j=1,2, \ldots, n$ :

$$
\begin{aligned}
\text { PayOff }_{j j} & =\text { Min } f_{j}(x) \\
x & \in X .
\end{aligned}
$$

where $x^{j, *}$ as the optimal solution and $\operatorname{Pay} O f f_{j j}=f_{j}\left(x^{j, *}\right)$ as the optimal value of the $j$ th objective is saved. Then, the optimal value of the $i$ th objective can be obtained by finding the optimal value of each objective $(j=1,2, \ldots, n ; j \neq i)$, as follows:

$$
\begin{gathered}
\text { PayOf } f_{i j}=\operatorname{Min} f_{i}(x) \\
f_{j}(x)=\text { PayOf } f_{j j} \\
x \in X \\
j \neq i,
\end{gathered}
$$

where $x^{i, j, *}$ and PayOff ${ }_{j j}=f_{i}\left(x^{i, j, *}\right)$ are the optimal solution and optimal value, belonging to the $i$ th objective. Accordingly, the payoff matrix is obtained as follows:

$$
\text { PayOff }=\left[\text { PayOf } f_{i j}\right] .
$$

After determining the payoff matrix for each objective $(i=1,2, \ldots, n)$, the following items are defined:

$$
\begin{gathered}
\operatorname{Min}\left(f_{i}\right)=\operatorname{Min}_{j}\left\{\text { PayOff }_{i j}\right\}=\text { payOff }_{i i} . \\
\operatorname{Max}\left(f_{i}\right)=\operatorname{Max}_{j}\left\{\text { PayOff }_{i j}\right\} . \\
R\left(f_{i}\right)=\operatorname{Max}\left(f_{i}\right)-\operatorname{Min}\left(f_{i}\right)
\end{gathered}
$$

The $R\left(f_{i}\right)$ value is used to normalize the objectives in MODM objective function. Supposed that the following values are obtained using the objectives payoff matrices:

$F_{1}$ : the minimum value of the first objective (optimal).

$\overline{F_{1}}$ : the minimum value of the first objective.

$F_{2}$ : the minimum value of the second objective (optimal).

$\overline{\overline{F_{2}}}$ : the minimum value of the second objective.

$F_{3}$ : the minimum value of the third objective (optimal).

$\overline{\overline{F_{3}}}$ : the minimum value of the third objective.

$F_{4}$ : the minimum value of the fourth objective (optimal).

$\overline{\overline{F_{4}}}$ : the minimum value of the fourth objective. 
Next, using the GP approach, the MODM model can be stated in terms of a single-objective one, as follows:

$$
\left\{\begin{array}{l}
\text { GP for MOMILP } \\
\min Z \\
Z \geq \frac{Z^{E 1}-\underline{F_{1}}}{\overline{F_{1}}-\underline{F_{1}}} \\
Z \geq \frac{Z^{S 1}-\underline{F_{2}}}{\overline{F_{2}}-\underline{F_{2}}} \\
Z \geq \frac{Z^{S 2}-\underline{F_{3}}}{\overline{F_{3}}-\underline{F_{3}}} \\
Z \geq \frac{Z^{S 3}-\underline{F_{4}}}{\overline{F_{4}}-\underline{F_{4}}} \\
x \in X=\text { MOMILP Constraints }=\operatorname{Eqs}(6)-(34) .
\end{array}\right.
$$

It should be mentioned that, after employing the GP approach to make a trade-off between different objectives, the final multi-objective model is a mixed-integer linear programming model, which could be solved using CPLEX Solver in GAMS.

\section{Practical implementation}

The first term of this section describes a practical case study, and the second term addresses collected input data. Finally, the proposed formulations are implemented, and the results are drawn in the third term of this section. Moreover, according to the main parameters, some sensitivity analyses are conducted, and some managerial insights are presented. The proposed model is coded in GAMS (General Algebraic Modelling System) and solved by the CPLEX solver on a personal computer with Intel Core i5 8400 processor, 8 GB RAM, and SSD $120 \mathrm{~GB}$.

\subsection{The case study}

A real case study in Iran is investigated in this section. The conducted studies from August 2012 to August 2013 in 148 countries show that Iran is one of the countries where corneal donation and corneal transplant surgery have been widely developed relative to its per capita population in recent years (Gain et al. 2016).

The central eye bank of Iran (CEBI) is a centralized national organization located in Tehran. The CEBI is mentioned as the largest transplantable corneal supplier globally in recent studies (Farazdaghi 2020). The CEBI provides patients' needs across the country (as the only legal entity) over the past 3 decades, only from a single city, Tehran, the capital, with approximately 9 million people (Kanavi et al. 2007; Javadi et al. 2020). According to the published report from the performance of the 3 past decades of the CEBI, the number of $55 \times 10^{-6} \pm 27 \times 10$ corneal transplant surgeries are performed each year, on average. In addition, there has been an increasing trend in providing PCT in Iran (Javadi et al. 2020). Figure 4 demonstrates the corneal donation process in Iran. 


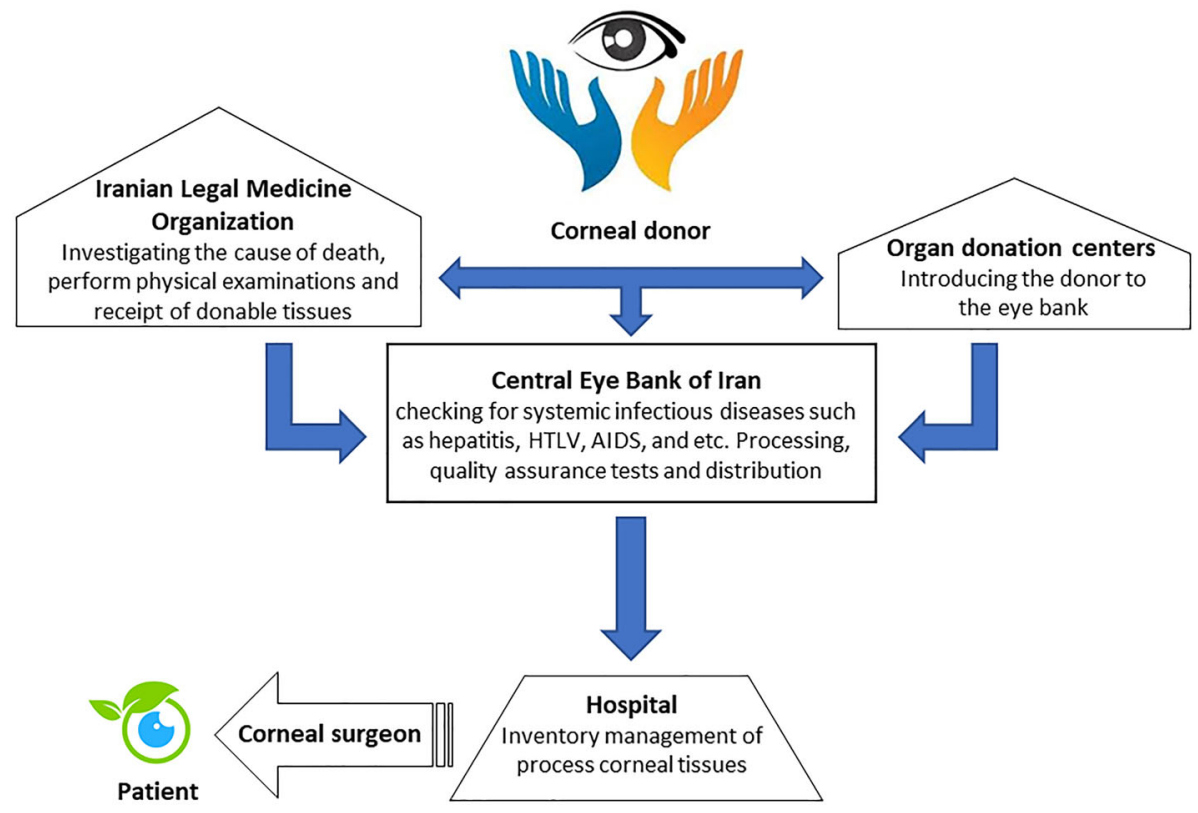

Fig. 4 Corneal transplantation in Iran

The investigated case study is a tertiary referral hospital (super specialty hospital) in Tehran's eye care, the first private ophthalmology center in Iran, including four sub-specialty ophthalmology clinics. The hospital inventory management team is responsible for policymaking, planning, and controlling the PCT inventory required for the hospital and its subspecialty clinics.

\subsection{Data collection}

The needed data are collected according to the CEBI statistical reports, the hospital inventory management team, experts' opinions, and related studies to this research. The hospital surgeons only request "good" to "excellent" qualities for PCT to perform the surgery based on the patients' medical condition. The ordered PCT could be stored for up to 7 days. Tables 8 , 9 and 10 illustrate the parameter values of the proposed model for the case study.

\subsection{Computational results and discussion}

In this section, the validation of the proposed model and its performance in the singleand multi-objective environments are investigated. The summary of the obtained results is inserted in Table 3. In this regard, the proposed model is first solved four times. Each comprises only one of the considered objectives in terms of a single-objective problem. Then, the multi-objective model is solved using a GP approach, and finally, the obtained results are discussed. Moreover, some sensitivity analyses are conducted on the main parameters to assess the proposed multi-objective model's performance and help decision-makers (DMs) find proper solutions for the problem. 
Table 3 Result summary of the single- and multi-objective models

\begin{tabular}{lrrrrr}
\hline Objective & \multicolumn{1}{c}{ E1-SO } & \multicolumn{1}{c}{ S1-SO } & \multicolumn{1}{c}{ S2-SO } & \multicolumn{1}{c}{ S3-SO } & \multicolumn{1}{c}{ MO } \\
\hline E1 & $848,900.000$ & $1,729,010.172$ & $981,030.200$ & $978,710.187$ & $974,559.392$ \\
S1 & 882.000 & 0.000 & 882.000 & 875.000 & 497.141 \\
S2 & 8.300 & 13.600 & 0.200 & 8.500 & 7.666 \\
S3 & 0.429 & 1.857 & 8.143 & 0.000 & 0.000 \\
MO & & & & & 0.564 \\
\hline
\end{tabular}

\subsubsection{Results and sensitivity analysis}

In this research, a MOMILP model is proposed to handle the inventory problem and then solved using a GP approach. The payoff matrix should be first obtained to utilize the GP approach in making a trade-off between different objectives. Using the obtained results, as already explained in Sect. 4.4, the objective functions' value is attained for the multi-objective model.

Table 3 summarizes the results of solving the proposed model as single- and multiobjective models. In addition, the values of objectives in the proposed multi-objective model are obtained using the GP approach. The single- and multi-objective models are addressed by SO and MO, respectively. As already pointed out, the current problem comprises four health-economic objectives. One of which deals with economic issues. The rest of the other three ones are concerned with health equity matters related to healthcare services. The first objective computes the total cost of the system related to inventory management. The second, third, and fourth objectives deal with the shortage, substitution rate, and product freshness, respectively.

Table 3 includes the optimal solution of each objective obtained by solving the proposed single- and multi-objective models. The proposed multi-objective model yields the best ideal solution when all objectives are equally important. Figure 5 illustrates a comparison between the obtained results of solving single- and multi-objective models. MO shows the ideal values of the different objective functions in the multi-objective model.

Figure 6 demonstrates the order quantity accompanied by a shortage imposed on the system in a 30-day planning horizon for single- and multi-objective models. More product units are ordered during the planning horizon to obtain the lowest shortage in the multi-objective model. The results show 98 shortage amounts, which occurred in the case of single-objective minimization of shortage (first health objective) for all demands totally, during the planning horizon. In this case, all the shortened items are compensated by ordering the eye bank through expedited service expeditiously. Therefore, by imposing the expedited service penalty costs, all demands are satisfied in the single-objective minimization of shortage. In this case, the amount of shortage reaches zero. No order is performed using expedited service for the rest of the other single- or multi-objective models, and the unfulfilled demands are lost.

Table 4 shows the substituted values at each of the ULs to meet the differentiated demand. For the current problem under consideration, product substitution only happens in the case of shortage. The substitution is not allowed at the UL-1, and the corresponding value for all models is zero. On the other hand, at the UL-3, the lost shortage is allowed. In this regard, with consideration of the relative importance of specific product availability at UL-3 $\left(\alpha_{p 3}\right)$, the substitution amount is determined by the model. However, the model is designed in a way 


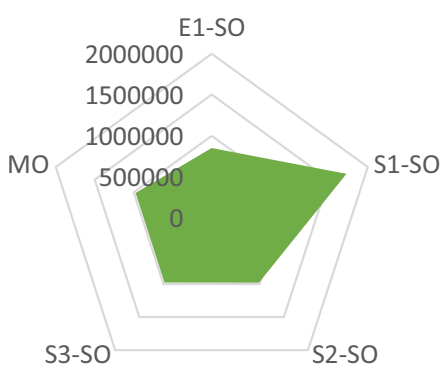

Value of economic objective

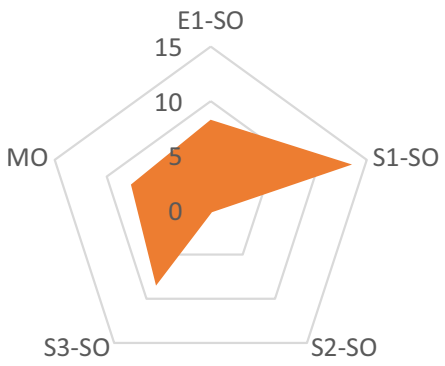

Value of substitution objective

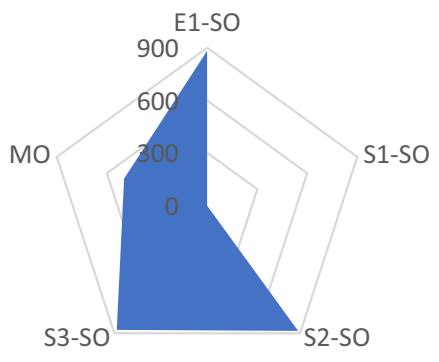

Value of shortage objective

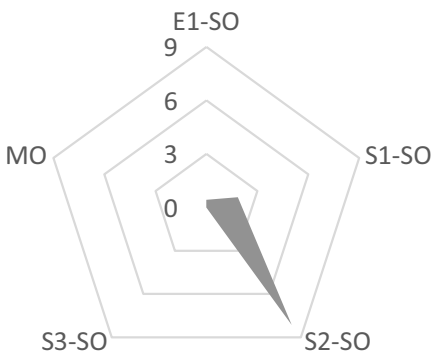

- Value of non-freshness objective

Fig. 5 Comparison of obtained values for four health-economic objectives by solving single-objective models

Fig. 6 Comparison of obtained solutions for order quantity and shortage by solving single- and multi-objective models

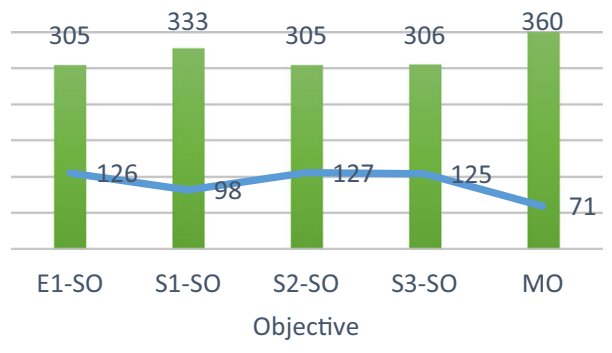

Order quantity

Shortage

Table 4 Amount of substitute product units for single- and multi-objective models

\begin{tabular}{lllll}
\hline & $u=1$ & $u=2$ & $u=3$ & Sum \\
\hline E1-SO & 0 & 83 & 0 & 83 \\
S1-SO & 0 & 112 & 9 & 121 \\
S2-SO & 0 & 2 & 0 & 2 \\
S3-SO & 0 & 85 & 0 & 85 \\
MO & 0 & 58 & 6 & 64
\end{tabular}


that prevents the substitution to a possible extent. Table 4 addresses 64 substituted product units obtained by solving the multi-objective model. It is the best ideal substitution amount compared to the results of the single-objective economic and health equity models (except the obtained optimum solution from the second health objective, i.e., substitution minimizing). If shortage minimization is the only objective in the model, the maximum substitution amount would be obtained.

Figure 7 depicts the total inventory assignment planning for satisfying the total demands at different ULs in terms of single- and multi-objective models. Figure 7 also shows how the substitution is planned for all ULs. As noticed before, solving the proposed multi-objective model yields the least amount of substitution compared to the other single-objective models except the second health objective (substitution minimization). In Fig. 7, the substitution objective addresses how the system satisfies demands when the system's only goal is to minimize the amount of product substitution and meet the different demands through the normal assignment as much as possible. The results indicate that the substitution objective has the lowest possible substitution value, zero (optimal value). In this case, all system demands are met normally.

The corresponding single-objective models to cost minimization and product freshness have relatively equal performance in meeting the demands using the product substitution strategy. Note that at the UL-1, due to risk factors for surgery failure and rejection, the
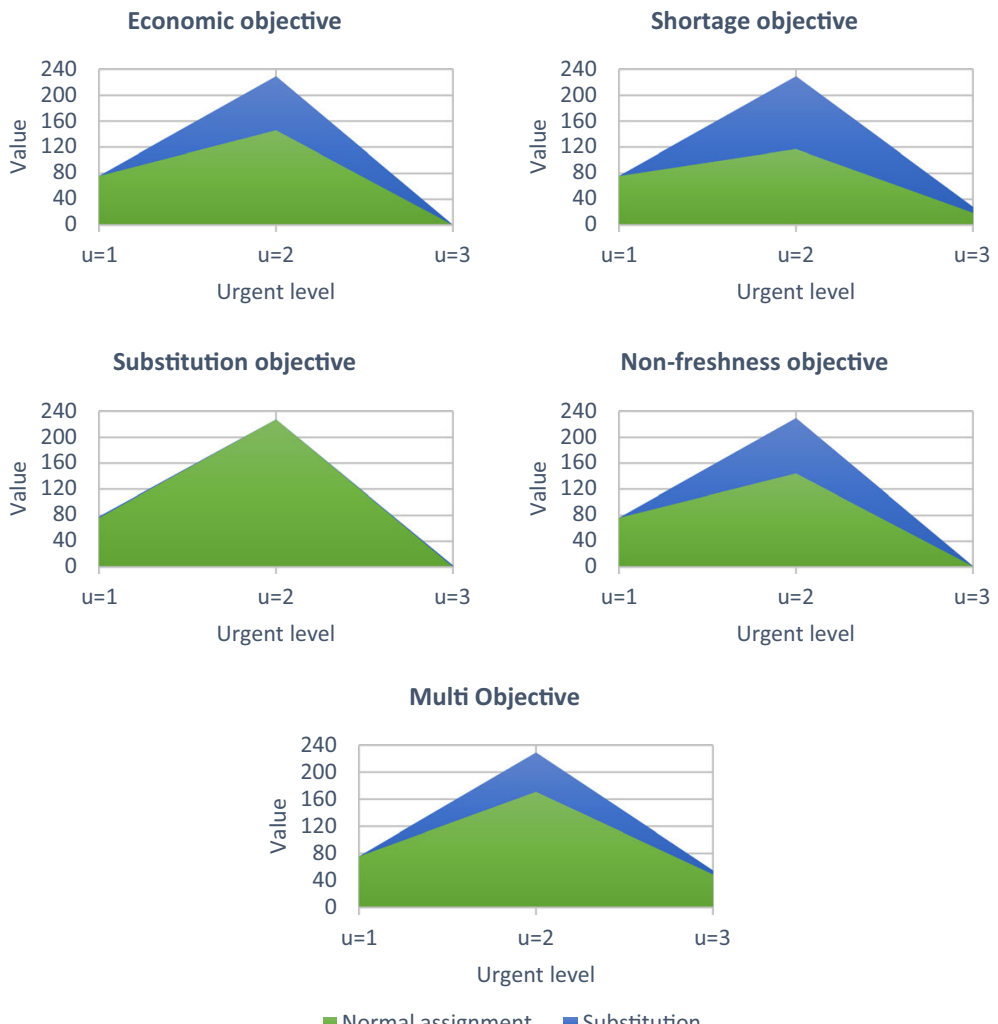

Fig. 7 How to assign inventory to different levels of demand urgency 
product substitution is not allowed and is equal to zero. Given that, it is not suggested to transship the items to the supplier as much as possible. However, it should not be neglected for real case situations. The proposed model is designed in such a way as to consume the inventory before its expedition threshold, if possible. Solving the proposed model verifies that the inventory is planned so that the wastage and transshipment amount is optimal and equal to zero.

Figure 8 illustrates the on-hand inventory level of each product at the end of each period in terms of single- and multi-objective models. During a 30-day planning horizon, the total onhand inventory obtained by solving the economic, shortage, substitution, and non-freshness single-objective models are 265, 117, 170, and 41 products units, respectively. Moreover, the multi-objective model maintains 73 products units during the same period. The results show that the performance of the non-freshness objective in the aspect of holding inventories is the best since the lowest holding cost is imposed on the system. However, in this case, only the freshness of the products is essential, and the other performance measurements are neglected. Afterward, the multi-objective model leads to the best results considering all objectives. The results illustrate that the proposed model is practical for improving the system's performance.

Figure 9 shows a comparison between the freshness importance of the satisfaction of the demands in single- and multi-objective models. The multi-objective model tends to consume the ordered quantity received from the supplier as fresh as possible due to some medical risks. The best performance in terms of the product's consumption in a fresh way belongs to the single-objective model corresponding to the freshness (the third health objective), followed by the proposed multi-objective model. In comparison, the worst performance belongs to the single-objective model corresponding to minimizing the substitution (the second health objective).

The proposed multi-objective model of the current study is solved using a GP approach. The importance of all objectives is considered the same. Table 5 presents the effect of the health-economic objectives' weights on the obtained results. If the economic objective function's weight is reduced, the shortage will decrease as the inventory system's total costs increase. Moreover, the inventory non-freshness and substitution rate would increase.

Table 5 indicates that the weight reduction of the shortage objective function leads to a significant decrease in the inventory system total costs, an increase in shortage amount and consumption of older-age inventory, and a reduction in the substitution. By reducing the substitution objective function's weight, more substitution for demands satisfaction is allowed; while reducing the total system costs and the shortage. In addition, inventory nonfreshness increases significantly. Despite increasing the weight of products, the freshness objective function has no significant effect on the system's total costs and shortage. However, the consumption of the older-age inventory increases, reducing the substitution amount to an acceptable level.

Figure 10 demonstrates the effect of purchasing fixed cost on the order quantity and shortage based on the proposed multi-objective model. The fixed purchasing cost affects the shortage. The higher the purchasing fixed cost, the lower the number of ordering periods, and the lower the order quantity in each period. This matter increases the shortage of inventories. On the other hand, the lower the order's fixed cost, the higher the number of orders. As shown in Fig. 10, the purchasing fixed costs and the shortage are inversely proportional. Due to the limited supplier's capacity and the hospital's storage capacity, if the fixed ordering costs exceed 20,000 monetary units, all available capacities are used to hold and order the item units in demand, if needed. In addition, the order quantity and shortage amounts would remain constant as the storage capacity is filled. 


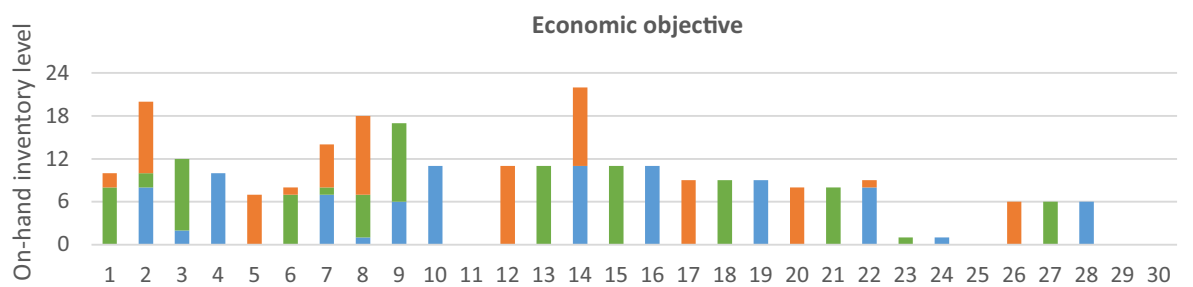
Planning horizon
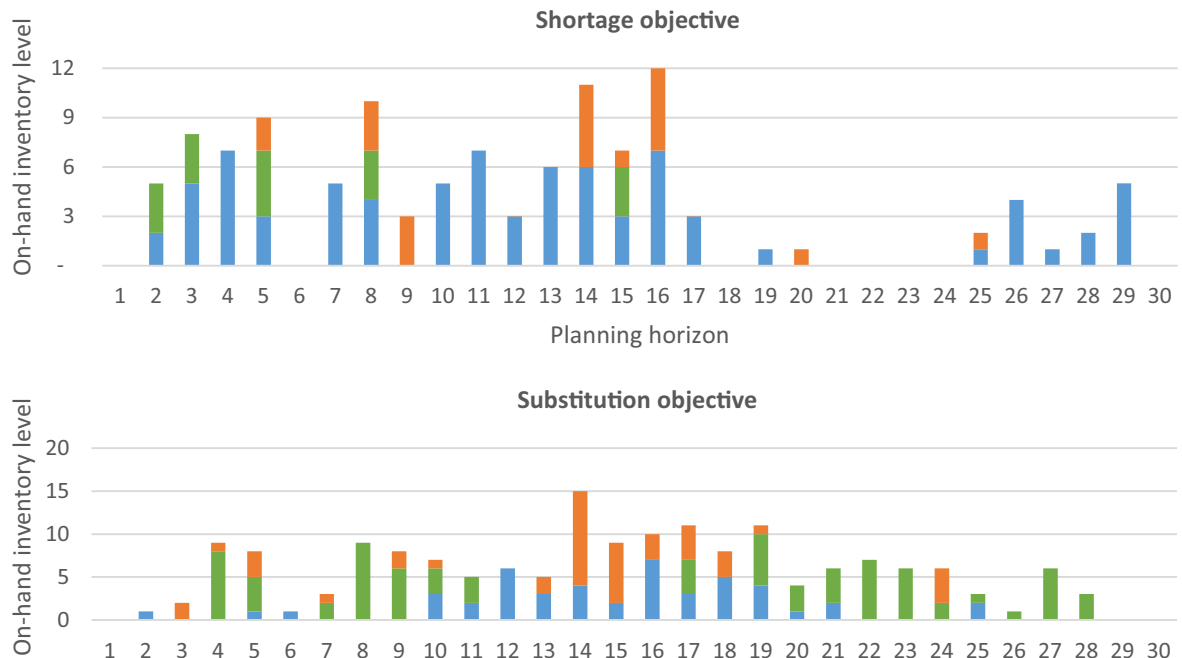
Planning horizon
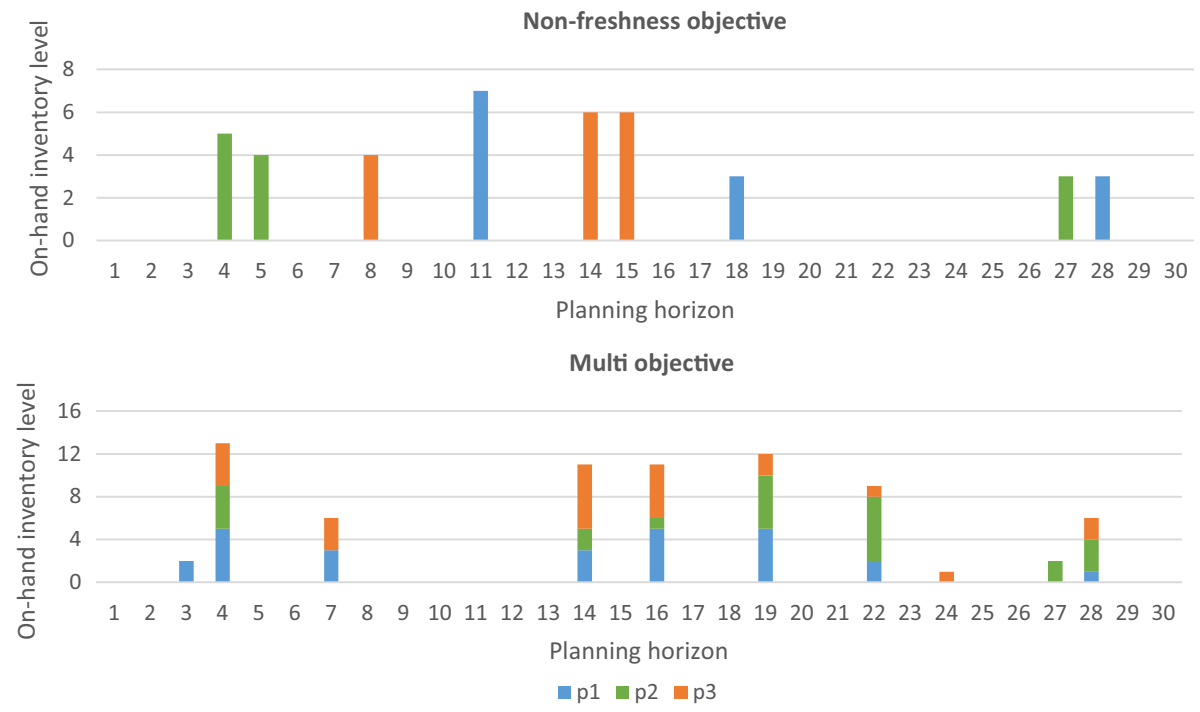

Fig. 8 On-hand inventory level at the end of each period 
Fig. 9 Comparison of the freshness of the assigned product for single- and multi-objective models

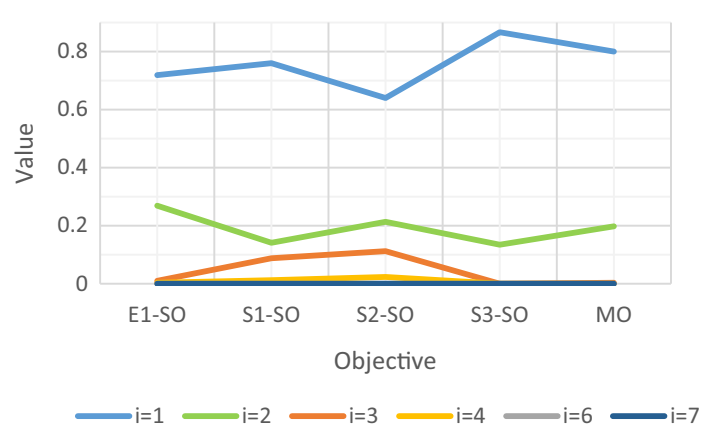

Table 5 Results of changing the health-economic objectives' weights on the optimal solution of the multiobjective model

\begin{tabular}{llllllll}
\hline$w=1$ & $w=2$ & $w=3$ & $w=4$ & $E 1$ & $S 2$ & $S 1$ & $S 3$ \\
\hline 0.7 & 0.1 & 0.1 & 0.1 & 848,900 & 882 & 8.2 & 0.571 \\
0.5 & 0.3 & 0.1 & 0.1 & $888,264.733$ & 755.2 & 9.4 & 0.571 \\
0.5 & 0.1 & 0.3 & 0.1 & $848,900.395$ & 882 & 7.8 & 0.571 \\
0.5 & 0.1 & 0.1 & 0.3 & $848,900.397$ & 882 & 6.6 & 0.571 \\
0.4 & 0.2 & 0.2 & 0.2 & $852,069.836$ & 869.313 & 8.881 & 0.429 \\
0.3 & 0.3 & 0.3 & 0.1 & $974,559.392$ & 497.141 & 5.3 & 0 \\
0.3 & 0.3 & 0.2 & 0.2 & $974,559.395$ & 497.141 & 11.498 & 0 \\
0.3 & 0.3 & 0.1 & 0.3 & $974,559.394$ & 497.141 & 15 & 0 \\
0.3 & 0.3 & 0.1 & 0.3 & $974,559.392$ & 497.141 & 15 & 0 \\
0.3 & 0.1 & 0.3 & 0.3 & 848,900 & 882 & 6.677 & 0.571 \\
0.3 & 0.1 & 0.3 & 0.3 & 848,900 & 882 & 6.677 & 0.571 \\
0.2 & 0.5 & 0.2 & 0.1 & $1,080,908.41$ & 220.557 & 8.502 & 0 \\
0.2 & 0.5 & 0.1 & 0.2 & $1,080,908.061$ & 220.556 & 16 & 0 \\
0.2 & 0.4 & 0.2 & 0.2 & $1,061,414.906$ & 270.724 & 8.349 & 0 \\
0.2 & 0.2 & 0.5 & 0.1 & $975,987.607$ & 497.869 & 3.071 & 0.571 \\
0.2 & 0.2 & 0.4 & 0.2 & $975,225.955$ & 497.481 & 3.814 & 0.428 \\
0.2 & 0.2 & 0.2 & 0.4 & $974,559.394$ & 497.141 & 6.9 & 0 \\
0.2 & 0.2 & 0.1 & 0.5 & $974,559.391$ & 497.141 & 15.331 & 0 \\
0.1 & 0.7 & 0.1 & 0.1 & $1,136,432.673$ & 82.816 & 8.939 & 0 \\
0.1 & 0.5 & 0.2 & 0.2 & $1,125,420.398$ & 114.82 & 4.426 & 0 \\
0.1 & 0.3 & 0.4 & 0.2 & $1,100,511.253$ & 187.13 & 2.164 & 0 \\
0.1 & 0.3 & 0.3 & 0.3 & $1,098,129.426$ & 186.725 & 2.879 & 0 \\
0.1 & 0.1 & 0.7 & 0.1 & $983,799.207$ & 501.854 & 1.105 & 1.714 \\
0.1 & 0.1 & 0.1 & 0.7 & $974,559.006$ & 497.141 & 7.666 & 0 \\
\hline & & & & & & & \\
\hline
\end{tabular}


Fig. 10 Results of changing the fixed purchasing cost (oc) on the normal order quantity and shortage of the multi-objective model

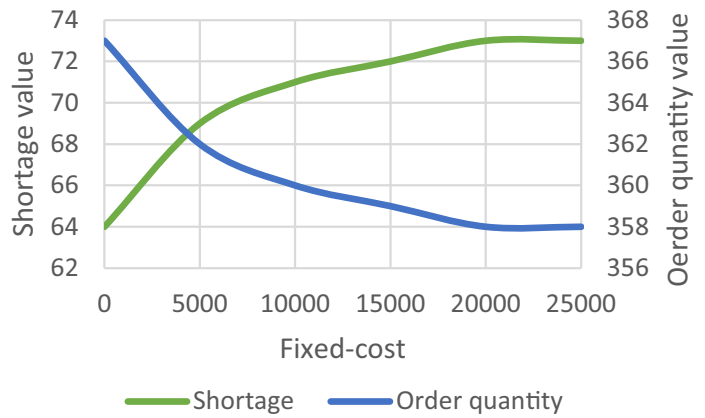

The proposed model is designed so that the products' availability at the UL- 1 and UL-2 is of great importance, respectively. If the inventory system at these two levels faces a shortage of demand satisfaction, the required item units would be supplied immediately by imposing the cost of expedited services. In case the constraints (23) and (24) are relaxed, only the value of $\alpha_{p u}$ affects the importance of product availability and the shortage of the two first levels of medical urgency. In this case, the obtained solution does not necessarily lead to satisfying the unfulfilled demand through expedited services in the case of shortage. The effect of the value of $\alpha_{p 3}$ parameter, on the solution of the proposed multi-objective model, is investigated considering all the products. The obtained results are reported in Table 6 and Fig. 11.

Table 5 shows that depending on the product's type and the corresponding value of the variable purchasing cost, there is less tendency to replenish. In this case, the model tends to accept the product units' shortage at the UL-3. Note that the lost shortage is allowed at the UL-3. In this case, the substitution with the other available products considering medical preferences may increase.

As the importance of product availability decreases, the tendency to use older-age available products increases. It should be noted that, by reducing the importance of product availability at a specific level, the numerical value of the shortage objective function may reduce due to $\alpha_{p u}$ parameter weight in the shortage objective function formula. Suppose the inventory system is not sensitive to product availability. In that case, it faces the most shortage amounts but

Table 6 Results of changing the product availability importance $\left(\alpha_{p u}\right)$ at UL-3 on the solution of the multiobjective model during the planning horizon

\begin{tabular}{llllllll}
\hline $\begin{array}{l}(p=1, p= \\
2, p=3)\end{array}$ & $E 1$ & $S 1$ & $S 2$ & $S 3$ & $\sum_{p, t} Q_{p t}$ & $\sum_{p, u, t} B_{p u t}$ & $\sum_{p, u, t} W_{p u t}$ \\
\hline$(10,10,10)$ & $972,674.429$ & 717.622 & 8.600 & 0 & 359 & 70 & 0 \\
$(5,10,10)$ & $947,654.668$ & 596.977 & 5.6 & 0 & 349 & 82 & 0 \\
$(0,10,10)$ & $935,812.421$ & 426.672 & 6.3 & 0.428 & 344 & 83 & 0 \\
$(10,5,10)$ & $935,121.8$ & 681.576 & 7.5 & 0.571 & 341 & 90 & 0 \\
$(10,0,10)$ & $927,404.547$ & 494.947 & 9.4 & 0.429 & 339 & 92 & 0 \\
$(10,10,5)$ & $961,517.164$ & 600.085 & 12.171 & 0.428 & 351 & 80 & 0 \\
$(10,10,0)$ & $942,920.993$ & 473.452 & 7.6 & 0.429 & 345 & 86 & 0 \\
$(5,5,5)$ & $972,674.429$ & 358.811 & 8.600 & 0 & 359 & 72 & 0 \\
$(0,0,0)$ & $1,793,930.17$ & 0 & 8.500 & 0 & 306 & 125 & 125 \\
\hline
\end{tabular}



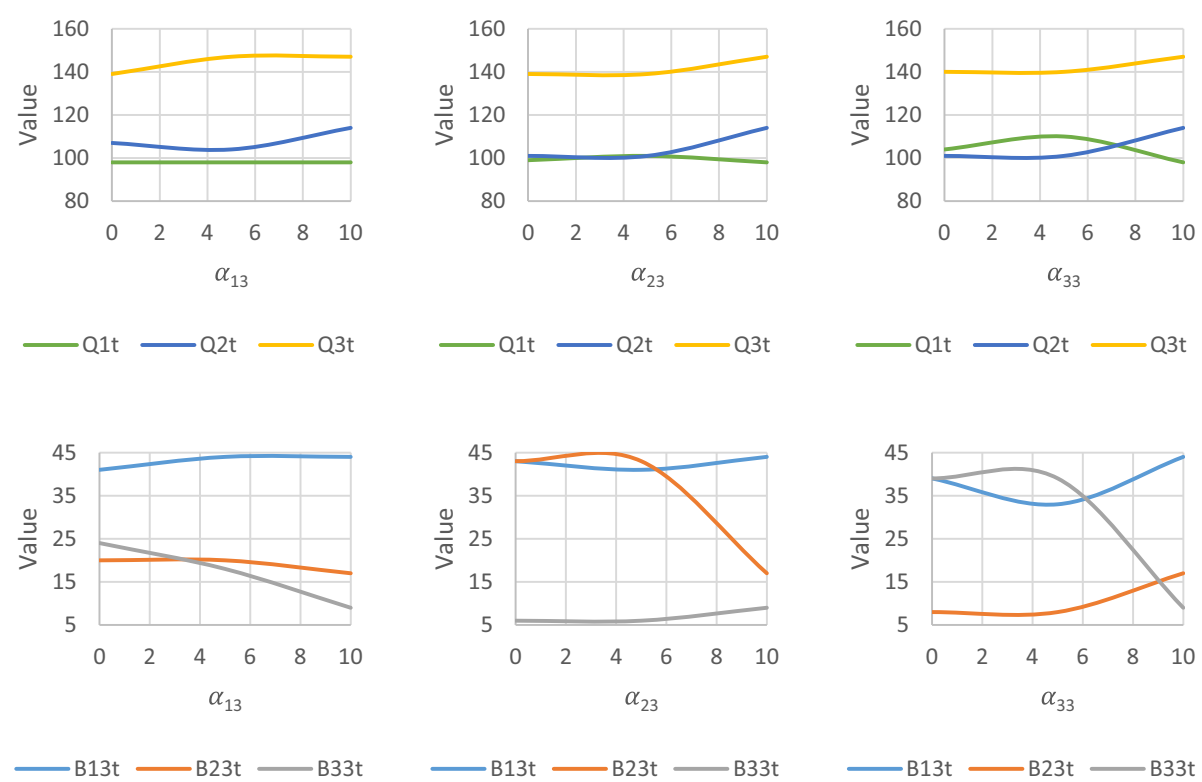

Fig. 11 Results of changing the product availability importance $\left(\alpha_{p u}\right)$ at UL-3 on the total order quantity and total shortage of the multi-objective model

Table 7 Results of changing the expedited service cost $\left(b c_{p t}\right)$ on the solution of the multi-objective model during the planning horizon

\begin{tabular}{llllllll}
\hline$b c_{p t}\left({ }^{*} p c_{p t)}\right.$ & $E 1$ & $S 1$ & $S 2$ & $S 3$ & $\sum_{p, t} Q_{p t}$ & $\sum_{p, u, t} B_{p u t}$ & $\sum_{p, u, t} W_{p u t}$ \\
\hline 0 & $848,900.4$ & 689.928 & 6.3 & 0.571 & 305 & 126 & 27 \\
0.5 & $872,203.7$ & 665.94 & 7.5 & 0.429 & 305 & 126 & 31 \\
1 & $903,761.6$ & 627.676 & 6.3 & 0.571 & 305 & 126 & 36 \\
1.5 & $938,583.2$ & 597.834 & 5.5 & 0.571 & 344 & 87 & 2 \\
1.6 & $941,476.8$ & 589.899 & 7.3 & 0.571 & 345 & 86 & 2 \\
2 & $951,803.3$ & 559.854 & 6.504 & 0 & 351 & 80 & 0 \\
2.5 & $963,856.4$ & 526.637 & 5.923 & 0 & 356 & 75 & 0 \\
3 & $974,559.4$ & 497.141 & 7.666 & 0 & 360 & 71 & 0 \\
3.5 & 984,127 & 470.773 & 7.259 & 0 & 364 & 67 & 0 \\
4 & $992,904.6$ & 447.14 & 6.895 & 0 & 367 & 64 & 0 \\
4.5 & $1,001,158$ & 425.9 & 6.567 & 0 & 370 & 61 & 0 \\
5 & $1,008,662$ & 406.587 & 6.269 & 0 & 373 & 58 & 0 \\
\hline
\end{tabular}

compensates the unfulfilled demands through the expedited services. Figure 11 demonstrates a more detailed comparison of the product's total order quantity based on the importance of their availability and total shortage amounts at each level of urgency in a 30-day planning horizon. 
Fig. 12 Comparison of shortage and substitution amounts regarding the expedited service cost $\left(b c_{p t}=n * p c_{p t}\right)$ increasing in the multi-objective model

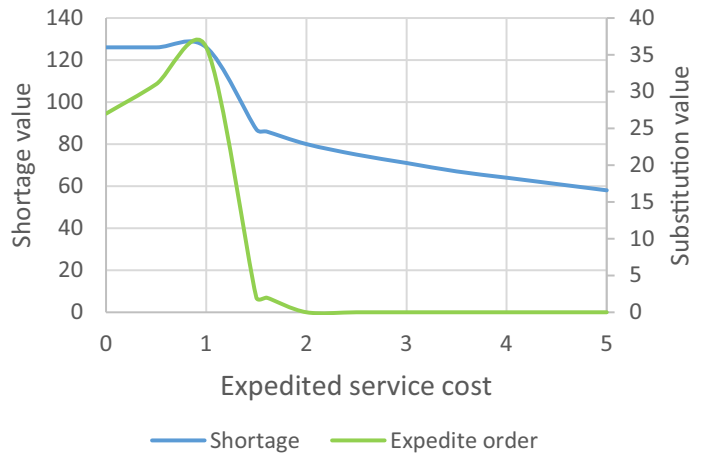

Table 7 addresses the effect of the expedited service cost (to compensate for the shortage) on the multi-objective model solution. Increasing the expedited service cost affects the system's total costs related to inventory management and lost shortage. The expedited service penalty usually is high. As long as the expedited service cost is less than 1.6 times the variable purchasing cost, the model tends to meet the shortage of product units by receiving the expedited services. Henceforth, as the expedited service cost increases, the model prefers to order more quantities to face fewer shortage amounts and avoid using the expedited services as much as possible. Figure 12 shows the compensated shortage by receiving the expedited orders considering the expedited service cost increases.

\subsubsection{Managerial insights}

Several managerial insights are presented in this section to provide a correct understanding of the usefulness of the proposed model and its solution to help healthcare managers. First, the proposed model helps assign PCT resources among demands with different priority levels, considering health equity as one of the pillars of healthcare systems. Health equity is the main criterion of providing healthcare services, especially in human organ transplants, due to the shortage, limited supply resources, and insufficient donors availability. The findings show that it is required to make a trade-off between health-economic objective functions. In this respect, while the model pursues to optimize multiple health objectives to meet the health equity, it avoids imposing additional costs on the system. Hence, the model provides an optimal economic solution without any weakening or negative impact on service levels.

Moreover, several extensive sensitivity analyses have been conducted on some critical parameters, and the performance of the proposed model has been evaluated by a real case study. Establishing the trade-off between health-economic objectives in the proposed model leads to achieving a satisfactory medical service level. The DMs select each objective function's importance in real cases, depending on the resources' availability and preferences. In this regard, a sensitivity analysis has been conducted on the effect of objectives weights on the model performance and obtained solution, which provides a wide view for DMs (Table 5). In human organ transplantation, the impact of promoting administrators' awareness and supporting them in making appropriate health-economic decisions is an issue that requires more efficient considerations. In this sense, providing a well-planned PCT inventory management system is of great significance. The supply chain researchers' efforts in creating 
motivation in human organ transplantation contribute significantly to public health improvement. Especially for the eye corneal tissues, it leads to the independence of countries from the PCT imports.

\section{Conclusion and future research}

This research aims to present an effective inventory model for managing the crucial SoHO products with limited shelf-lives by focusing on health equity. In this paper, a trade-off between health equity and economic objectives of healthcare systems is provided by a practical MOMILP model. Besides, the priority differentiation of demands is considered. We have considered an optimal periodic review policy for determining order quantity and replenishment periods. To determine which demand should be met first, they are classified into three levels based on medical urgency: 'High', 'Medium', and 'Low'. Patient demands at each UL have the same features and are differentiated from other demand levels. The emergency demands are included in "High" urgent level. Hence, in this survey, several demand-oriented heuristic policies are extended to optimally satisfy the demands and reduce the risk of lives as well as minimize the total shortage and wastage of the system. We have developed a hybrid issuance policy to minimize wastage according to the requirements of demands at each urgent level.

The present study goes beyond the typical HSC studies for the perishable medical items (which have often been performed on drugs and blood products) by researching the realworld concerns of PCT's inventory management. This research can be enumerated as the first-ever applicable study related to the inventory management of the human transplantable organs, which expands the boundaries of the operational research studies on the HSC and promotes society's health by supporting the healthcare DMs.

The proposed model's performance is validated by a real case study of the ESC. The results show that the model provides an optimal health-economic solution for satisfying the demands with different priorities. By implementing the proposed model for the real-life case, the results show that appropriate heuristic policies are adopted for each demand urgency based on medical conditions. In this regard, the same priority demands receive the same services equitably. For demand satisfaction at UL-1 and UL-2, the shortage is zero, and for UL-3 is determined optimally. The model also reduces the unnecessary substitutions as much as possible. Moreover, inventory management is done by the proposed model in such a way that, in most cases, the product units are consumed freshly for patient treatment. Therefore, the proposed model improves the performance of the healthcare system where there are severe risks to the patient's health in aspects of health equity. On the other hand, the results show that the proposed model minimizes wastage and decreases the system's operational costs, leading to improving the system's economic performance. 
In summary, the presented model helps the SoHO product administrators manage the systems inventory so that minimum shortage and wastage occur without weakening the service level concerning health equity. Moreover, several sensitivity analyses have been conducted on some critical parameters, which provide wide managerial insights for healthcare DMs.

More investigation on the ESC as the most abundant and successful allogeneic transplantation in the world with operational research approaches could be a future stream. To do so, one can study the ESC as a network or at the different echelon of the chain, such as the eye banks and local distribution centers, by employing other modeling techniques and solution methods. In this regard, surveys on improving the sustainability of SoHO supply chain are among the highly encouraged cases neglected so far. Besides, emergency conditions and unplanned demands disrupt the efficiency of the medical service delivery systems. Providing mathematical models and practical solutions, as another future study, to deal with emergency conditions is highly encouraged. Developing the present model to handle realworld uncertainties and encountering disastrous situations such as the Covid-19 pandemic considering health equity to achieve the desired services level is another interesting topic for future research.

Funding This research did not receive any specific grant from funding agencies in the public, commercial, or not-for-profit sectors.

\section{Appendix. Parameter values}

See Tables 8, 9 and 10 . 


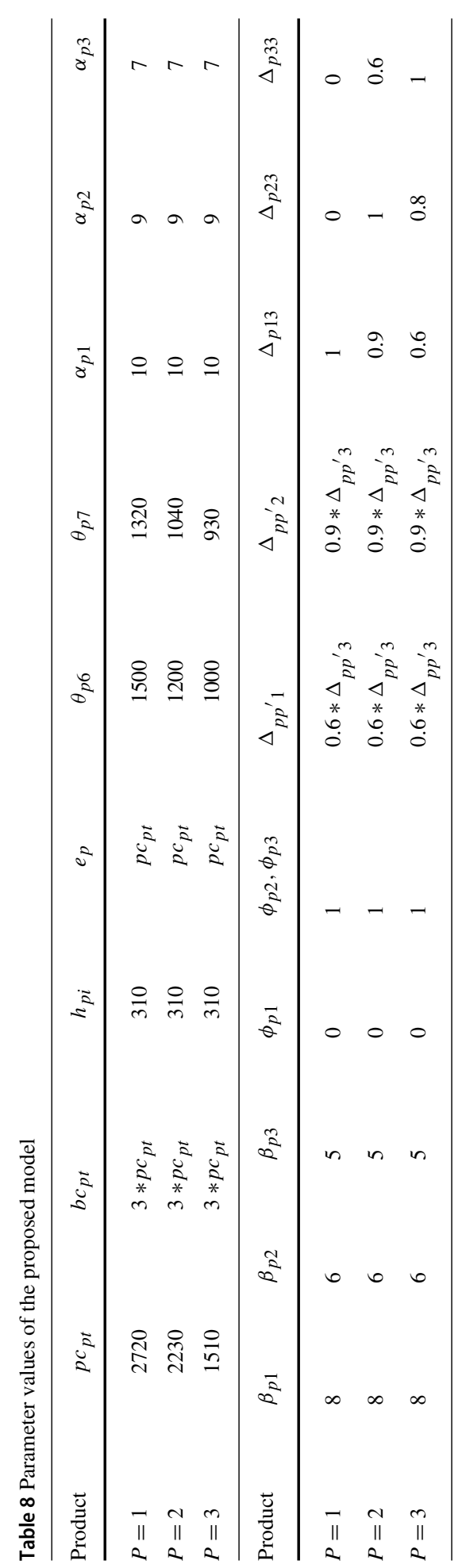


Table 9 Products substitution allowance based on medical priorities

\begin{tabular}{llll}
\hline $\mathrm{S}_{p p^{\prime}}$ & $P^{\prime}=1$ & $P^{\prime}=2$ & $P^{\prime}=3$ \\
\hline$P=1$ & 1 & 1 & 1 \\
$P=2$ & 0 & 1 & 1 \\
$P=3$ & 0 & 1 & 1 \\
\hline
\end{tabular}

\begin{tabular}{ll}
\hline Parameter & Value \\
\hline$T$ & $1,2, \ldots, 30$ \\
$R$ & $1,2, \ldots, 30$ \\
$P$ & $\{1,2,3\}$ \\
$U$ & $\{1,2,3\}$ \\
$I$ & 7 \\
$T r$ & 6 \\
$M A_{p}$ & 6 \\
$v o l$ & 45 \\
$\gamma_{p}$ & 1 \\
$m v_{p}$ & 15 \\
$o c$ & 9000 \\
dem $_{p 1 t}$ & $\sim U(0,2)$ \\
dem $_{p 2 t}$ & $\sim U(1,4)$ \\
dem $_{p 3 t}$ & $\sim U(0,3)$ \\
cap $_{p t}$ & $\sim U(4,11)$ \\
\hline
\end{tabular}

Table 10 Other parameter values of the proposed model

\section{References}

Al Mahmasani L, Hodroj MH, Finianos A, Taher A (2021) COVID-19 pandemic and transfusion medicine: the worldwide challenge and its implications. Ann Hematol 100:1115-1122

Assessment RR (2020) Coronavirus disease 2019 (COVID-19) in the EU/EEA and the UK-ninth update. Eur Cent Dis Prev Control Stock

Attari MYN, Abdoli N (2020) Reducing wastages and shortages of blood products at hospitals with considering $\mathrm{ABO} / \mathrm{Rh}$ (D) compatibility. J Ind Eng Decis Mak 1:24-48

Balarajan Y, Selvaraj S, Subramanian SV (2011) Health care and equity in India. Lancet 377:505-515

Benjamin GC (2020) Ensuring health equity during the COVID-19 pandemic: the role of public health infrastructure. Rev Panam Salud Publica. 44:e70

Bhattacharya B, Lam F (2020) Overcoming shortages of essential medicines: perspectives from industrial and systems engineering and public health practice. In: Smith K, Ram P (eds) Transforming global health. Springer, Cham

Braveman P (2006) Health disparities and health equity: concepts and measurement. Annu Rev Public Heal 27:167-194

Chaudhary V, Kulshrestha R, Routroy S (2018) State-of-the-art literature review on inventory models for perishable products. J Adv Manag Res 15(3)

Cheraghi S, Hosseini-Motlagh S-M (2018) Responsive and reliable injured-oriented blood supply chain for disaster relief: a real case study. Ann Oper Res 291:129-167

Civelek I, Karaesmen I, Scheller-Wolf A (2015) Blood platelet inventory management with protection levels. Eur J Oper Res 243:826-838

Culyer AJ (2001) Equity-some theory and its policy implications. J Med Ethics 27:275-283

放 Springer $\int \mathrm{D} / \mathrm{W}$ 
Dehghani M, Abbasi B (2018) An age-based lateral-transshipment policy for perishable items. Int J Prod Econ 198:93-103

Derikvand H, Hajimolana SM, Jabbarzadeh A, Najafi SE (2020) A robust stochastic bi-objective model for blood inventory-distribution management in a blood supply chain. Eur J Ind Eng 14:369-403

Dillon M, Oliveira F, Abbasi B (2017) A two-stage stochastic programming model for inventory management in the blood supply chain. Int J Prod Econ 187:27-41

Evans G (2014) Biowaste and biological waste treatment. Routledge

Ezugwu AE, Olusanya MO, Govender P (2019) Mathematical model formulation and hybrid metaheuristic optimization approach for near-optimal blood assignment in a blood bank system. Expert Syst Appl 137:74-99

Farazdaghi M (2020) World's Largest Eye Bank has Served a Nation for Three Decades. J Ophthalmic vis Res 15:126

Franco C, Alfonso-Lizarazo E (2017) A structured review of quantitative models of the pharmaceutical supply chain. Complexity 2017

Gain P, Jullienne R, He Z et al (2016) Global survey of corneal transplantation and eye banking. JAMA Ophthalmol 134:167-173

Garcin T, Gauthier A, Crouzet E et al (2019) Innovative corneal active storage machine for long-term eye banking. Am J Transplant 19:1641-1651

Gunantara N (2018) A review of multi-objective optimization: methods and its applications. Cogent Eng 5:1502242

Gupta AM, Ojha S, Nagaraju P et al (2021) Impact of the novel coronavirus disease and lockdown on the packed red blood cells inventory management: an experience from a tertiary care oncology center in Western India. Hematol Transfus Cell Ther 43:126-132

Habibi-Kouchaksaraei M, Paydar MM, Asadi-Gangraj E (2018) Designing a bi-objective multi-echelon robust blood supply chain in a disaster. Appl Math Model 55:583-599

Hosseinifard Z, Abbasi B (2018) The inventory centralization impacts on sustainability of the blood supply chain. Comput Oper Res 89:206-212

Hosseini-Motlagh S-M, Samani MRG, Cheraghi S (2020a) Robust and stable flexible blood supply chain network design under motivational initiatives. Socioecon Plan Sci 70:100725

Hosseini-Motlagh S-M, Samani MRG, Homaei S (2020b) Blood supply chain management: robust optimization, disruption risk, and blood group compatibility (a real-life case). J Ambient Intell Humaniz Comput 11:1085-1104

Hosseini-Motlagh S-M, Samani MRG, Homaei S (2020c) Toward a coordination of inventory and distribution schedules for blood in disasters. Socioecon Plan Sci, p 100897

Javadi MA, Kanavi MR, Safi S (2020) A 27-Year Report from the Central Eye Bank of Iran. J Ophthalmic vis Res 15:149

Jones D, Tamiz M (2016) A review of goal programming. In: Greco S, Ehrgott M, Figueira J (eds) Multiple criteria decision analysis. International series in operations research \& management science, vol 233. Springer, New York, NY

Kanavi MR, Javadi MA, Sanagoo M (2007) Indications for penetrating keratoplasty in Iran. Cornea 26:561-563

Larimi NG, Yaghoubi S (2019) A robust mathematical model for platelet supply chain considering social announcements and blood extraction technologies. Comput \& Indus Eng 137:106014

Liu W, Ke GY, Chen J, Zhang L (2020) Scheduling the distribution of blood products: A vendor-managed inventory routing approach. Transp Res Part E Logist Transp Rev 140:101964

Malhotra K, Tank G, Patel K et al (2018) A study to evaluate the keratoanalyzer in success of penetrating kertoplasty. Indian J Clin Exp Ophthalmol 4:465-468

Manrodt K, Schott D (2016) Health care supply chain management: elements, operations, and strategies. Jones $\&$ Bartlett Learning

Mosadegh H, Khakbazan E, Salmasnia A, Mokhtari H (2017) A fuzzy multi-objective goal programming model for solving an aggregate production planning problem with uncertainty. Int J Inf Decis Sci 9:97-115

Mousazadeh M, Torabi SA, Pishvaee MS, Abolhassani F (2018) Accessible, stable, and equitable health service network redesign: A ROBUST mixed possibilistic-flexible approach. Transp Res Part E Logist Transp Rev 111:113-129

Najafi M, Ahmadi A, Zolfagharinia H (2017) Blood inventory management in hospitals: considering supply and demand uncertainty and blood transshipment possibility. Oper Res Heal Care 15:43-56

Osorio AF, Brailsford SC, Smith HK (2018) Whole blood or apheresis donations? A multi-objective stochastic optimization approach. Eur J Oper Res 266(1):193-204

Panda A, Vanathi M, Kumar A et al (2007) Corneal graft rejection. Surv Ophthalmol 52:375-396

Pirabán A, Guerrero WJ, Labadie N (2019) Survey on blood supply chain management: Models and methods. Comput Oper Res 112:104756 
Puranam K, Novak DC, Lucas MT, Fung M (2017) Managing blood inventory with multiple independent sources of supply. Eur J Oper Res 259(2):500-511

Rajendran S, Ravindran AR (2017) Platelet ordering policies at hospitals using stochastic integer programming model and heuristic approaches to reduce wastage. Comput Ind Eng 110:151-164

Rajendran S, Ravindran AR (2019) Inventory management of platelets along blood supply chain to minimize wastage and shortage. Comput Ind Eng 130:714-730

Rajendran S, Srinivas S (2020) Hybrid ordering policies for platelet inventory management under demand uncertainty. IISE Trans Healthc Syst Eng 10:113-126

Regidor E, Martínez D, Calle ME et al (2008) Socioeconomic patterns in the use of public and private health services and equity in health care. BMC Health Serv Res 8:183

Rosenwasser GO, Szczotka-Flynn LB, Ayala AR et al (2017) Effect of cornea preservation time on success of Descemet stripping automated endothelial keratoplasty: a randomized clinical trial. JAMA Ophthalmol 135:1401-1409

Safaei AS, Heidarpoor F, Paydar MM (2018) Group purchasing organization design: a clustering approach. Comput Appl Math 37:2065-2093

Saha E, Ray PK (2018) Inventory management and analysis of pharmaceuticals in a healthcare system. In: Ray P, Maiti J (eds) Healthcare systems management: methodologies and applications. Managing the Asian century. Springer, Singapore

Samani MRG, Hosseini-Motlagh S-M, Ghannadpour SF (2019a) A multilateral perspective towards blood network design in an uncertain environment: methodology and implementation. Comput Ind Eng 130:450-471

Samani MRG, Hosseini-Motlagh SM (2019b) An enhanced procedure for managing blood supply chain under disruptions and uncertainties. Ann Oper Res 283(1):1413-1462

Santhi G, Karthikeyan K (2016) Recent review article on pharmaceutical inventory models. Int J Pharm Tech Res 9:435-443

Shamsoddin E (2020) Substantial aspects of health equity during and after COVID-19 pandemic: a critical review. Int Netw Gov Sci Advice

Sohrabi M, Zandieh M, Nadjafi BA (2021b) Dynamic demand-centered process-oriented data model for inventory management of hemovigilance systems. Healthc Inform Res 27:73-81

Sohrabi M, Zandieh M, Afshar-Nadjafi B (2021a) An equity-oriented multi-objective inventory management model for blood banks considering the patient condition: a real-life case. Sci Iran

Uthayakumar R, Karuppasamy SK (2019) An EOQ model for deteriorating items with different types of time-varying demand in healthcare industries. J Anal 27(1):3-18

van Roode T, Pauly BM, Marcellus L et al (2020) Values are not enough: qualitative study identifying critical elements for prioritization of health equity in health systems. Int J Equity Health 19:1-13

Williams KA, Coster DJ (2007) The immunobiology of corneal transplantation. Transplantation 84:806-813

Zare M, Javadi MA, Einolahi B et al (2011) Indications for corneal transplantation at Labbafinejad medical center. Bina J Ophthalmol 16:306-311

Zhang S, Meng F (2020) Analysis of the consistency and consensus for group decision-making with intervalvalued intuitionistic fuzzy preference relations. Comput Appl Math 39:1-35

Publisher's Note Springer Nature remains neutral with regard to jurisdictional claims in published maps and institutional affiliations. 